\title{
Obesity and inflammation: the effects of weight loss
}

\author{
L. Kirsty Forsythe, Julie M. W. Wallace and M. Barbara E. Livingstone* \\ Northern Ireland Centre for Food and Health, Centre for Molecular Biosciences, \\ University of Ulster, Coleraine BT52 1SA, UK
}

\begin{abstract}
Following the discovery of TNF- $\alpha$ and leptin as secretory products of adipocytes in the early 1990s, subsequent obesity research focused on the new functional role of adipose tissue, as an active endocrine organ. Many more inflammatory peptides have been linked to adiposity, which ultimately characterised obesity as a state of low-grade systemic inflammation, or 'metaflammation' which may link obesity to its co-morbidities. The aim of the present review is to examine the effects of weight loss on inflammation in overweight and obese, but otherwise healthy, populations. Studies were broadly classified into four types (diet, physical activity, diet and physical activity combined, and surgical interventions) and discussed according to the method used to induce weight loss. All studies measured at least one obesity-related inflammatory marker (ORIM). The overall finding from the present review is that weight loss does improve inflammation in terms of both the inflammatory (C-reactive protein, TNF- $\alpha$, IL- 6 and leptin) and anti-inflammatory (adiponectin) ORIM. Within this, the greatest improvements in ORIM are observed in studies achieving a weight loss of at least $10 \%$. However, a number of methodological issues have been identified as potential limitations within the literature including the sex and age of subjects, sample size, study duration and the assessment of body composition. In conclusion, although a period of weight loss per se is capable of reversing the unfavourable inflammatory profile evident in the obese state, further studies are required to determine the time needed, in which a reduced weight is maintained, in order to benefit from improved inflammatory status long term.
\end{abstract}

Obesity: Adipose tissue: Inflammation: Weight-loss interventions

\section{Introduction}

The global epidemic of obesity is a major public health problem, which is driven largely by its co-morbidities including insulin resistance, type 2 diabetes, CVD and cancer. Recent data from the UK show that approximately two-thirds of the population are classified as overweight or obese (defined as having a BMI $\geq 25.0 \mathrm{~kg} / \mathrm{m}^{2}$ ) ${ }^{(1)}$ and that over the last decade, the proportion of individuals classified as normal weight (BMI $\left.18.5-24.9 \mathrm{~kg} / \mathrm{m}^{2}\right)^{(1)}$ is slowly declining $^{(2)}$. Consequently, being overweight has become the 'norm' in today's society ${ }^{(3)}$ and it has been estimated, based on current rates, that by $2050,60 \%$ of males and $50 \%$ of females in the UK will be obese (BMI $\left.\geq 30 \cdot 0 \mathrm{~kg} / \mathrm{m}^{2}\right)^{(4)}$.

In 1993, Hotamisligil et al. ${ }^{(5)}$ discovered that cells of the adipose tissue (adipocytes) in rodents secreted TNF- $\alpha$, a pro-inflammatory cytokine and, although unknown to them, this discovery was to shape the future of obesity research worldwide $^{(6,7)}$. Following the discovery of cytokine-like leptin in 1994 by Zhang et al. ${ }^{(8)}$, it became clear that adipose tissue could no longer be regarded as an inert storage organ, but rather it is an active endocrine organ ${ }^{(8,9)}$. Subsequent research has demonstrated that adipose tissue is involved in many metabolic and physiological pathways including inflammation, insulin sensitivity and vascular haemostasis ${ }^{(10,11)}$. Since these initial discoveries, many more inflammatory peptides have been linked to adiposity, for example: the anti-inflammatory peptide hormone, adiponectin $^{(12)}$; acute-phase proteins, such as C-reactive protein (CRP) and pro-inflammatory cytokines including IL-6 ${ }^{(13)}$ and to date, more than fifty adiposity-related inflammatory molecules have been identified ${ }^{(14,15)}$.

Consequently, obesity is now characterised as a state of chronic or low-grade systemic inflammation ${ }^{(13,16-18)}$. More recently, the term 'metaflammation' has been used to describe the metabolically triggered inflammation ${ }^{(19)}$, due to the abnormal circulating levels of inflammatory molecules evident in the obese state (Fig. 1) ${ }^{(7,20-23)}$.

Abbreviations: CRP, C-reactive protein; FFM, fat-free mass; FM, fat mass; LCD, low-calorie diet; ORIM, obesity-related inflammatory marker; SAT, subcutaneous adipose tissue; VAT, visceral adipose tissue; VLCD, very-low-calorie diet.

* Corresponding author: Professor Barbara Livingstone, fax +44 287032 3059, email mbe.livingstone@ulster.ac.uk 

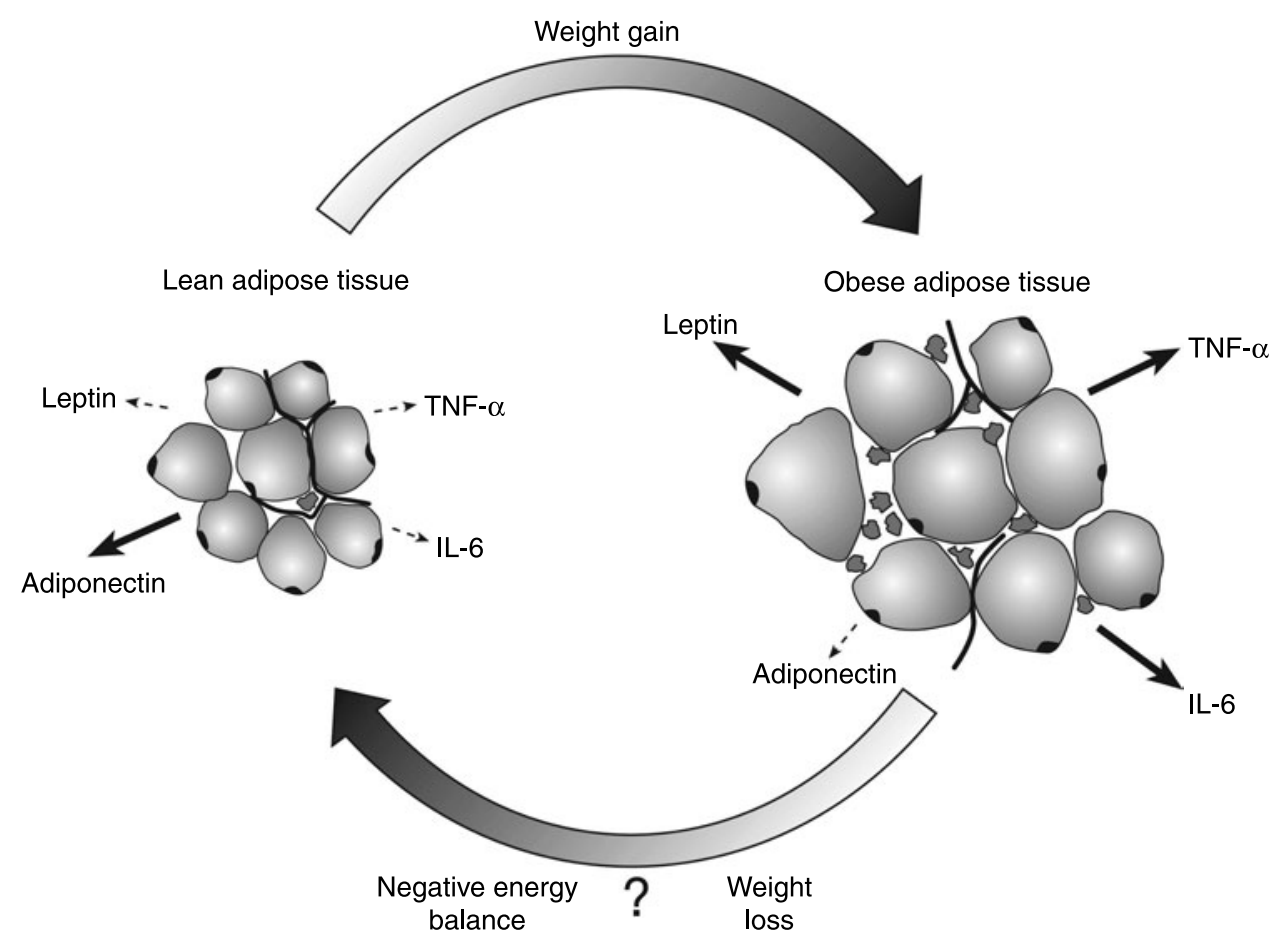

Fig. 1. During weight gain, cells of the adipose tissue increase in size due to the storage of excess lipids (adipocyte hypertrophy), which disrupts normal cellular function. Consequently, abnormal circulating levels of inflammatory molecules are evident in the obese state and obesity is recognised as a state of chronic or low-grade systemic inflammation, specifically characterised by an increase in the obesity-related inflammatory markers (such as leptin, TNF- $\alpha$ and IL-6) and a decrease in the anti-inflammatory marker, adiponectin. Research has shown that a period of weight loss can improve this unfavourable inflammatory state; however, it is likely that this is mainly due to a negative energy balance in the short term, rather than decreasing adiposity.

Furthermore, several researchers have also demonstrated metabolic differences between the different adipose tissue depots, that is, subcutaneous adipose tissue (SAT) and visceral adipose tissue (VAT), with VAT (or central adiposity) in particular being associated with an increased risk of co-morbidities such as diabetes and $\mathrm{CVD}^{(24-29)}$ which may partly be attributed to differences in the secretions of inflammatory molecules from each fat depot ${ }^{(30,31)}$.

It is widely accepted that the abnormal inflammatory profile observed in obesity is due to adipocyte hypertrophy (increased size), caused by the storage of the excess lipids (Fig. 1 $^{(15,31-33)}$. In addition, some research indicates that an increase in the number of adipocytes (hyperplasia) may also contribute to the abnormal inflammatory profile; however, evidence for this view is less consistent in the literature $^{(34,35)}$. Researchers have recently been criticised for taking an 'over-simplistic' view of adipose tissue ${ }^{(36)}$ in failing to take account of other cells residing in the tissue, such as macrophages, pre-adipocytes and mast cells, and their potential contribution to the inflammatory profile observed in obesity ${ }^{(37-39)}$. Indeed, by definition, an adipokine is a protein secreted by the adipocytes ${ }^{(14)}$; however, adipocytes are not the source of all inflammatory molecules in the obese state. For example, CRP is released from the liver ${ }^{(40)}$ and therefore is not, by definition, an adipokine. However, it is beyond the scope of the present review to discuss the molecular mechanisms and the various cellular sources of each inflammatory molecule involved in the development of obesity-related inflammation. Therefore, all inflammatory molecules associated with obesity will be referred to hereafter as obesity-related inflammatory markers (ORIM).

Along with the discovery of the secretion of TNF- $\alpha$ from adipose tissue, Hotamisligil et al. ${ }^{(5)}$ also demonstrated a link between adiposity, TNF- $\alpha$ and insulin resistance, and inflammation is now well understood as a link between obesity and its clustering of metabolic abnormalities and co-morbidities ${ }^{(5,41-43)}$. Given the evidence that elevated levels of ORIM could be causal in the development of its associated co-morbidities, many researchers have hypothesised that the reduction in disease risk seen in response to weight loss could be as a result of an improved inflammatory profile, that is, a decrease in the pro-inflammatory molecules, such as CRP, and an increase in the antiinflammatory molecule, adiponectin (Fig. 1). To our knowledge, reviews in this area have solely focused on $\mathrm{CRP}$ as an independent risk factor for $\mathrm{CVD}^{(44-46)}$. Therefore, the aim of the present review is to examine the effects of weight loss on a number of ORIM in overweight and obese, but otherwise healthy, populations.

\section{Weight loss and inflammation}

Weight-loss interventions reviewed in the present paper ( $n$ 66) were broadly classified into four types according to the method of weight loss employed; diet, physical activity, diet and physical activity combined, and surgical interventions. In order to examine these effects in apparently healthy populations (with the exception of gastric surgery 
interventions), studies including insulin-resistant or diabetic subjects, those with any cardiovascular risk factor or patients suffering from any other chronic disease were excluded. All studies included at least one study group aiming for weight loss and measured at least one ORIM. In those studies where different methods of weight loss were compared, for example, diet $v$. diet and physical activity combined; each study group is discussed below according to the method used.

\section{Dietary interventions}

Studies using dietary modification alone (Table 1;

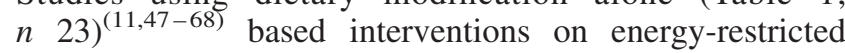
diets ( $n$ 5), low-, or very-low-calorie diets (LCD or VLCD, respectively) ( $n$ 8), or low-fat diets ( $n$ 4). Some also compared the effects of diets with different macronutrient compositions ( $n$ 6), for example, low-fat $v$. low-carbohydrate, although in the majority, an energy deficit was the ultimate cause of weight loss. The duration of the interventions ranged from $2 \mathrm{~d}$ to 14 months and the number of participants ranged from seven to eighty-three. Based on mean baseline BMI values reported for each study group, $15 \%$ were carried out in overweight subjects $(25.0-$ $\left.29.9 \mathrm{~kg} / \mathrm{m}^{2}\right), 67 \%$ in obese $\left(30 \cdot 0-39.9 \mathrm{~kg} / \mathrm{m}^{2}\right)$ and $9 \%$ in morbidly obese subjects $\left(\geq 40 \cdot 0 \mathrm{~kg} / \mathrm{m}^{2}\right)$. Two studies did not report baseline BMI.

Overall, studies using LCD as the method of intervention resulted in the highest mean weight loss, compared with those using other methods, such as low-fat diets (10 v. $5 \%$, respectively). The greatest improvements in the serum or plasma concentrations of ORIM were observed in these studies where a weight loss of at least $10 \%$ was reported $^{(51,54-56,59,68)}$. For example, Kasim-Karakas et al. failed to show any significant changes in serum CRP, IL-6 or adiponectin with an $8 \%(6 \mathrm{~kg})$ weight loss after a 12 -month low-fat diet ${ }^{(62)}$, whereas in the two LCD studies which reported a weight loss of approximately $15 \%$ (15 and $19 \mathrm{~kg}$, respectively), a $32 \%$ decrease in plasma CRP after 14 months $^{(56)}$ and a $24 \%$ decrease in plasma IL-6 after only 6 months ${ }^{(55)}$ was observed. No LCD study measured adiponectin concentration after weight loss; however, a shorter-term (6 weeks) VLCD reporting an $11 \%(11 \mathrm{~kg})$ weight loss was associated with a $19 \%$ increase in serum $\operatorname{adiponectin}^{(59)}$. Furthermore, the benefits of losing more than $10 \%$ weight, rather than more modest weight loss, are evident when similar dietary methods of weight loss are employed. For example, You et al. demonstrated that a $5 \%$ $(5 \mathrm{~kg})$ weight loss was unable to induce significant improvements in any ORIM measured ${ }^{(47)}$. On the other hand, Bougoulia et al. demonstrated a 22, 21, 86 and $32 \%$ decrease in plasma CRP, TNF- $\alpha$, IL- 6 and leptin, respectively, and a $72 \%$ increase in plasma adiponectin concentrations with a $19 \%(20 \mathrm{~kg})$ weight $\operatorname{loss}^{(51)}$. It should be noted that these studies were carried out in females with differing menopausal status; however, the overall benefits of a weight loss greater than $10 \%$ can clearly be seen.

Nine studies included in the present review compared the effect of two or more different weight-loss diets on inflammation $^{(53,57,60,63-68)}$, with the majority $(n 8)$ lasting between 2 and 3 months. Hannum et al. failed to show a significant decrease in serum CRP with modest weight loss $(4-7 \%$ or $4-6 \mathrm{~kg})$ in either a self-selected or portioncontrolled LCD which were both low-fat $(20 \%$ of total energy $)^{(53)}$. A further study by Heald et al. carried out in females of a similar age reported that when high-fat foods were replaced with reduced-fat alternatives, this resulted in a small, but significant, weight loss $(2 \%$ or $1 \mathrm{~kg})$ and a significant decrease in serum CRP $(24 \%)^{(60)}$. Similar improvements in plasma and serum CRP concentrations were also reported in studies comparing diets of different macronutrient composition ${ }^{(63,64,67)}$. However, no differences were reported between the diets, in terms of their effects on weight loss or inflammation. In an additional two studies that compared the effects of low-fat and moderatefat diets, both designed for weight loss, significant reductions in plasma ${ }^{(65)}$ and serum ${ }^{(66)}$ leptin concentrations were observed in all intervention groups, but with no significant difference between diets observed. In contrast in another study, a low-glycaemic-load diet was associated with a significantly greater improvement in serum CRP concentration compared with that of a low-fat diet group (48 v. $5 \%$ ) despite the same percentage weight loss in both groups $^{(68)}$. Of note, the latter study was the only dietary comparison study to include both males and females.

Finally, Clément et al. ${ }^{(57)}$ examined the effects of a $2 \mathrm{~d}$ and $28 \mathrm{~d}$ VLCD on plasma leptin concentrations in eight and twenty-one obese females, respectively. The $2 \mathrm{~d}$ VLCD was only sufficient to induce an $11 \%$ decrease in plasma leptin concentration, compared with a $53 \%$ decrease observed in the $28 \mathrm{~d}$ VLCD group ${ }^{(57)}$. However, the decrease in leptin reported for the $2 \mathrm{~d}$ VLCD group was most probably attributed to the acute energy restriction rather than the degree of weight loss per se. Furthermore, those in the $2 \mathrm{~d}$ VLCD group had a considerably higher BMI, fat mass (FM) and plasma leptin concentration at baseline than those in the $28 \mathrm{~d}$ VLCD group, making it difficult to draw conclusions from the study.

In two of the above studies, the authors noted that significant improvements in ORIM (CRP and IL-6 in this case) were observed when results for the two study groups were pooled for analysis, implying an insufficient sample size to detect changes in these ORIM following weight loss in each study group ${ }^{(53,66)}$. This limitation was also noted by Pereira et al., albeit their results were significant ${ }^{(68)}$, and it was also evident in the study by Keogh et al. ${ }^{(48)}$. Their study initially aimed to investigate the effect of weight loss on two ORIM (CRP and adiponectin) in a low-carbohydrate ( $n$ 13) and high-carbohydrate (n 12) group. However, after a substantial drop-out, the small sample size led to results for the two intervention groups to be combined. Overall, a modest weight loss $(6 \%$ or $5 \mathrm{~kg}$ ) resulted in a $17 \%$ increase in circulating adiponectin concentration, with CRP remaining unchanged from baseline. However, this may have been due to the small sample size that remained ( $n 13)$, even after combining the two groups ${ }^{(48)}$.

\section{Physical activity interventions}

The duration of physical activity interventions included in the present review ranged from 2 months to 1 year and the number of participants ranged from eight to 199 


\section{NS Nutrition Research Reviews}

Table 1. Summary of dietary intervention studies investigating obesity-related inflammatory markers before and after weight loss (WL) by type of intervention and degree of weight loss $(n$ 23)

\begin{tabular}{|c|c|c|c|c|c|c|c|c|c|c|c|c|c|c|c|c|c|c|c|}
\hline \multirow[b]{2}{*}{ Study } & \multirow[b]{2}{*}{$\begin{array}{l}\text { Subjects } \\
\qquad(n)\end{array}$} & \multirow[b]{2}{*}{ Sex } & \multirow[b]{2}{*}{$\% \mathrm{M}$} & \multicolumn{2}{|c|}{ Age (years) } & \multirow[b]{2}{*}{$\begin{array}{l}\text { Intervention } \\
\text { group(s) }\end{array}$} & \multirow[b]{2}{*}{ Duration } & \multicolumn{5}{|c|}{ Baseline } & \multirow{2}{*}{$\begin{array}{c}\text { Body } \\
\text { composition } \\
\text { method(s) }\end{array}$} & \multirow[b]{2}{*}{$\% \mathrm{WL}$} & \multicolumn{4}{|c|}{$\%$ Decrease } & \multirow[b]{2}{*}{$\begin{array}{l}\% \text { Increase } \\
\text { in adiponectin }\end{array}$} \\
\hline & & & & Mean & Range & & & $\begin{array}{c}\mathrm{BMI} \\
\left(\mathrm{kg} / \mathrm{m}^{2}\right)\end{array}$ & $\begin{array}{l}\text { WC } \\
(\mathrm{cm})\end{array}$ & $\begin{array}{l}\text { Waist:hip } \\
\text { ratio }\end{array}$ & $\% \mathrm{BF}$ & $\begin{array}{l}\text { Fat mass } \\
(\mathrm{kg})\end{array}$ & & & CRP & TNF- $\alpha$ & IL-6 & Leptin & \\
\hline \multicolumn{20}{|l|}{ ERD } \\
\hline $\begin{array}{l}\text { You et al. } \\
(2004)^{(47) *}\end{array}$ & 17 & $\mathrm{~F}$ & 0 & 57 & $50-70$ & $\begin{array}{l}\text { ERD (to achieve WL } \\
\text { of } 0.5-1 \mathrm{~kg} / \text { week) }\end{array}$ & 6 months & $\sim$ & $\sim$ & 0.79 & 48.3 & $42 \cdot 7$ & $\mathrm{DXA}+\mathrm{CT}$ & $5 \cdot 2$ & NS & NS & NS & $\sim$ & $\sim$ \\
\hline $\begin{array}{l}\text { Keogh et al. } \\
(2007)^{(48) *}\end{array}$ & 13 & $M / F$ & $\sim$ & 49 & $\sim$ & $\begin{array}{l}\text { ERD (to achieve WL } \\
\text { of } 0.5-1 \mathrm{~kg} / \text { week) }\end{array}$ & 12 months & 32.9 & $\sim$ & $\sim$ & $\sim$ & $\sim$ & $\sim$ & 5.6 & NSt & $\sim$ & $\sim$ & $\sim$ & $17 \cdot 1$ \\
\hline $\begin{array}{l}\text { Davì et al. } \\
(2002)^{(49) *}\end{array}$ & 11 & $\mathrm{~F}$ & 0 & $\sim$ & $24-63$ & $\operatorname{ERD}(5.0 \mathrm{MJ} / \mathrm{d})$ & 12 weeks & $44.0 \ddagger$ & $\sim$ & $0.98 \ddagger$ & $\sim$ & $\sim$ & $\sim$ & $9 \cdot 2 \ddagger$ & $22 \cdot 2 † \ddagger$ & $\sim$ & $\sim$ & $33.3 \ddagger$ & $\sim$ \\
\hline $\begin{array}{l}\text { McLaughlin et al. } \\
(2002)^{(50) *}\end{array}$ & al. 18 & $\mathrm{~F}$ & 0 & 44 & $\sim$ & $\begin{array}{l}\text { ERD (to achieve WL } \\
\text { of } 0.5 \mathrm{~kg} / \text { week) }\end{array}$ & 3 months & $31 \cdot 4$ & 95.0 & $\sim$ & $\sim$ & $\sim$ & $\sim$ & 9.7 & NSt & $\sim$ & $\sim$ & $\sim$ & $\sim$ \\
\hline $\begin{array}{l}\text { Bougoulia et al. } \\
(2006)^{(51) *}\end{array}$ & 36 & $\mathrm{~F}$ & 0 & 35 & $\sim$ & $\operatorname{ERD}(\downarrow 2.5 \mathrm{MJ} / \mathrm{d})$ & 6 months & 38.5 & 108.5 & 0.90 & 42.5 & & BIA & $19 \cdot 2$ & $22.3 \dagger$ & 20.9 & 85.9 & 31.5 & $72 \cdot 2$ \\
\hline$\%$ WL mean & & & & & & & & & & & & & & 9.8 & & & & & \\
\hline \multicolumn{20}{|l|}{ LCD } \\
\hline $\begin{array}{l}\text { Heilbronn et al. } \\
(2001)^{(52)}\end{array}$ & 83 & $\mathrm{~F}$ & 0 & 48 & $\sim$ & $\begin{array}{l}\operatorname{LCD}(6.0 \mathrm{MJ} / \mathrm{d}+ \\
15 \% \mathrm{E} \text { from fat })\end{array}$ & 12 weeks & 33.8 & $98 \cdot 3$ & 0.83 & $\sim$ & $\sim$ & $\sim$ & $\sim$ & $25.9 \dagger$ & $\sim$ & $\sim$ & $\sim$ & $\sim$ \\
\hline $\begin{array}{l}\text { Hannum et al. } \\
(2004)^{(53) \star}\end{array}$ & 27 & $\mathrm{~F}$ & 0 & 37 & $24-56$ & $\begin{array}{l}\text { LCD (self-selected, } \\
5.7 \mathrm{MJ} / \mathrm{d})\end{array}$ & 8 weeks & $31 \cdot 6$ & 99.9 & $\sim$ & $40 \cdot 1$ & 34.6 & DXA & 4.2 & NSt & $\sim$ & $\sim$ & $\sim$ & $\sim$ \\
\hline $\begin{array}{l}\text { Hannum et al. } \\
(2004)^{(53) \star}\end{array}$ & 26 & $\mathrm{~F}$ & 0 & 38 & $24-55$ & $\begin{array}{l}\text { LCD (portion controlled, } \\
5.7 \mathrm{MJ} / \mathrm{d})\end{array}$ & & 31.8 & $100 \cdot 8$ & $\sim$ & $40 \cdot 3$ & 35.3 & DXA & 6.5 & NS & $\sim$ & $\sim$ & $\sim$ & $\sim$ \\
\hline $\begin{array}{l}\text { Considine et al. } \\
(1996)^{(54) *}\end{array}$ & 7 & $\mathrm{M} / \mathrm{F}$ & 14 & 37 & $\sim$ & $\begin{array}{l}\text { LCD (liquid-protein, } \\
3.4 \mathrm{MJ} / \mathrm{d} \text { ) }\end{array}$ & 10 weeks & $40 \cdot 4$ & $\sim$ & $\sim$ & $\sim$ & & $\sim$ & $10 \cdot 0$ & $\sim$ & $\sim$ & $\sim$ & $53 \cdot 0$ & $\sim$ \\
\hline $\begin{array}{l}\text { Brunn et al. } \\
(2003)^{(55) \star}\end{array}$ & 19 & M & 100 & 35 & $18-48$ & $\operatorname{LCD}(4 \cdot 2-6 \cdot 2 \mathrm{MJ} / \mathrm{d})$ & 24 weeks & $38 \cdot 7$ & 124.9 & $\sim$ & $\sim$ & $50 \cdot 3$ & DXA & 14.7 & $\sim$ & 29.2 & 24.4 & $\sim$ & $\sim$ \\
\hline $\begin{array}{l}\text { Tchernof et al. } \\
(2002)^{(56)}\end{array}$ & 25 & $\mathrm{~F}$ & 0 & 57 & $\sim$ & $\operatorname{LCD}(5.0 \mathrm{MJ} / \mathrm{d})$ & 14 months & $35 \cdot 2$ & $\sim$ & $\sim$ & $42 \cdot 4$ & $\sim$ & $\mathrm{DXA}+\mathrm{CT}$ & $15 \cdot 6$ & $32 \cdot 2$ & $\sim$ & $\sim$ & $\sim$ & $\sim$ \\
\hline \multirow{2}{*}{\multicolumn{20}{|c|}{ VLCD }} \\
\hline & & & & & & & & & & & & & & & & & & & \\
\hline $\begin{array}{l}\text { Clément et al. } \\
(2004)^{(57) \star}\end{array}$ & 8 & $\mathrm{~F}$ & 0 & 41 & $\sim$ & $\operatorname{VLCD}(\downarrow 2.7 \mathrm{MJ} / \mathrm{d})$ & $2 d$ & $46 \cdot 6$ & $\sim$ & $\sim$ & $\sim$ & 61.0 & DXA & 0.8 & $\sim$ & $\sim$ & $\sim$ & $11 \cdot 3$ & $\sim$ \\
\hline $\begin{array}{l}\text { Clément et al. } \\
(2004)^{(57) \star}\end{array}$ & 21 & $\mathrm{~F}$ & 0 & 39 & $\sim$ & $\operatorname{VLCD}(3.4 \mathrm{MJ} / \mathrm{d})$ & 4 weeks & 35.0 & $\sim$ & $\sim$ & $\sim$ & $46 \cdot 0$ & DXA & $6 \cdot 4$ & $\sim$ & $\sim$ & $\sim$ & 52.9 & $\sim$ \\
\hline $\begin{array}{l}\text { Bastard et al. } \\
(2000)^{(58) \star}\end{array}$ & 14 & $\mathrm{~F}$ & 0 & 45 & $28-64$ & $\operatorname{VLCD}(3.9 \mathrm{MJ} / \mathrm{d})$ & 3 weeks & 39.5 & $\sim$ & 0.96 & $\sim$ & $47 \cdot 0$ & DXA & $8.4^{\S}$ & NS & NSt & $16 \cdot 5$ & $46 \cdot 1$ & $\sim$ \\
\hline $\begin{array}{l}\text { Raitakari et al. } \\
(2004)^{(59)}\end{array}$ & 67 & $\mathrm{M} / \mathrm{F}$ & 30 & 46 & $\sim$ & $\operatorname{VLCD}(2.3 \mathrm{MJ} / \mathrm{d})$ & 6 weeks & 35.2 & 110.0 & 0.93 & $\sim$ & $\sim$ & $\sim$ & 10.9 & $42 \cdot 4 \dagger$ & $\sim$ & $\sim$ & $\sim$ & 18.8 \\
\hline \multicolumn{20}{|l|}{ LF diets } \\
\hline $\begin{array}{l}\text { Heald et al. } \\
(2004)^{(60) \star}\end{array}$ & 20 & $\mathrm{~F}$ & 0 & 45 & $\sim$ & $\begin{array}{l}\text { LF (replace HF for } \\
\text { LF foods) }\end{array}$ & 3 months & 30.0 & $\sim$ & $\sim$ & $35 \cdot 4$ & $\sim$ & $\mathrm{BIA}$ & NS & $\uparrow \mathrm{NS}+$ & $\sim$ & $\sim$ & $\sim$ & $\sim$ \\
\hline $\begin{array}{l}\text { Heald et al. } \\
(2004)^{(60) *}\end{array}$ & 20 & $\mathrm{~F}$ & 0 & 44 & $\sim$ & $\downarrow+$ LF (combination) & & 32.0 & $\sim$ & $\sim$ & 37.4 & $\sim$ & $\mathrm{BIA}$ & NS & $\uparrow \mathrm{NS} \uparrow$ & $\sim$ & $\sim$ & $\sim$ & $\sim$ \\
\hline $\begin{array}{l}\text { Heald et al. } \\
(2004)^{(60) *}\end{array}$ & 20 & $\mathrm{~F}$ & 0 & 39 & $\sim$ & $\begin{array}{l}\downarrow \text { Fat (reduced-fat } \\
\text { alternatives) }\end{array}$ & & $30 \cdot 4$ & $\sim$ & $\sim$ & 35.9 & $\sim$ & BIA & 1.7 & $24.3+$ & $\sim$ & $\sim$ & $\sim$ & $\sim$ \\
\hline $\begin{array}{l}\text { Brekke et al. } \\
(2005)^{(61) \star}\end{array}$ & 25 & $\mathrm{M} / \mathrm{F}$ & 60 & 44 & $25-55$ & $\mathrm{LF}(\leq 30 \% \mathrm{E}$ from fat) & 16 weeks & $25 \cdot 3$ & $89 \cdot 2$ & & $28 \cdot 0$ & $\sim$ & $\mathrm{BIA}$ & NS & $\sim$ & $\sim$ & $\sim$ & NS & NS \\
\hline $\begin{array}{l}\text { Rokling- } \\
\text { Andersen et al. } \\
(2007)^{(11) *}\end{array}$ & 45 & M & 100 & 45 & $\sim$ & $\begin{array}{l}\text { LF (current } \\
\quad \text { recommendations) }\end{array}$ & 1 year & $29 \cdot 2$ & $\sim$ & 1.00 & 24.5 & $23 \cdot 1$ & NIR & 4.4 & NS & $\uparrow 4.1$ & NS & $10 \cdot 3$ & $\downarrow 1.5$ \\
\hline
\end{tabular}




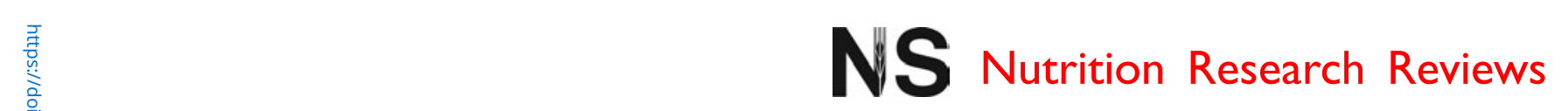

Table 1. Continued

\begin{tabular}{|c|c|c|c|c|c|c|c|c|c|c|c|c|c|c|c|c|c|c|c|}
\hline \multirow[b]{2}{*}{ Study } & \multirow[b]{2}{*}{$\begin{array}{l}\text { Subjects } \\
(n)\end{array}$} & \multirow[b]{2}{*}{ Sex } & \multirow[b]{2}{*}{$\% \mathrm{M}$} & \multicolumn{2}{|c|}{ Age (years) } & \multirow[b]{2}{*}{$\begin{array}{l}\text { Intervention } \\
\text { group(s) }\end{array}$} & \multirow[b]{2}{*}{ Duration } & \multicolumn{5}{|c|}{ Baseline } & \multirow{2}{*}{$\begin{array}{c}\text { Body } \\
\text { composition } \\
\text { method(s) }\end{array}$} & \multirow[b]{2}{*}{$\% \mathrm{WL}$} & \multicolumn{4}{|c|}{$\%$ Decrease } & \multirow[b]{2}{*}{$\begin{array}{l}\text { \% Increase } \\
\text { in adiponectin }\end{array}$} \\
\hline & & & & Mean & Range & & & $\begin{array}{c}\mathrm{BMI} \\
\left(\mathrm{kg} / \mathrm{m}^{2}\right)\end{array}$ & $\begin{array}{l}\text { WC } \\
(\mathrm{cm})\end{array}$ & $\begin{array}{l}\text { Waist:hip } \\
\text { ratio }\end{array}$ & $\% \mathrm{BF}$ & $\begin{array}{l}\text { Fat mass } \\
(\mathrm{kg})\end{array}$ & & & CRP & TNF- $\alpha$ & IL-6 & Leptin & \\
\hline $\begin{array}{l}\text { Kasim-Karakas } \\
\text { et al. }(2006)^{(62)}\end{array}$ & 20 & $\mathrm{~F}$ & 0 & 61 & $\sim$ & LF (15\% E from fat) & 12 months & $29 \cdot 1$ & $\sim$ & $\sim$ & $\sim$ & $\sim$ & $\sim$ & 7.9 & NSt & $\sim$ & NSt & $\sim$ & NS \\
\hline Mean \% WL & & & & & & & & & & & & & & 4.7 & & & & & \\
\hline \multicolumn{20}{|l|}{$\begin{array}{l}\text { Diet comparison } \\
\text { studies }\end{array}$} \\
\hline $\begin{array}{l}\text { O'Brien et al. } \\
(2005)^{(63) \star}\end{array}$ & 19 & $\mathrm{~F}$ & 0 & 44 & $\sim$ & LF (ER, 5.0 MJ/d) & 3 months & 33.6 & $\sim$ & $\sim$ & 41.4 & $\sim$ & DXA & 4.7 & $24 \cdot 1 \dagger \ddagger$ & $\sim$ & $\sim$ & $\sim$ & $\sim$ \\
\hline $\begin{array}{l}\text { O'Brien et al. } \\
(2005)^{(63) \star}\end{array}$ & 22 & $\mathrm{~F}$ & 0 & 44 & $\sim$ & $\begin{array}{l}\text { Low-carbohydrate } \\
\text { (Atkins, no ER) }\end{array}$ & & 33.6 & $\sim$ & $\sim$ & 41.4 & $\sim$ & DXA & $8 \cdot 3$ & $24 \cdot 1 \dagger \ddagger$ & $\sim$ & $\sim$ & $\sim$ & $\sim$ \\
\hline $\begin{array}{l}\text { Viguerie et al. } \\
(2005)^{(65) \star}\end{array}$ & 25 & $\mathrm{~F}$ & 0 & $\sim$ & $21-49$ & $\begin{array}{c}\mathrm{HF} \text { (42\% E from fat, } \\
\downarrow 2.5 \mathrm{MJ} / \mathrm{d})\end{array}$ & 10 weeks & $36 \cdot 1$ & $\sim$ & $\sim$ & $43 \cdot 4$ & 43.5 & $\mathrm{BIA}$ & $6 \cdot 7$ & $\sim$ & $\sim$ & $\sim$ & $31 \cdot 8$ & $\sim$ \\
\hline $\begin{array}{l}\text { Viguerie et al. } \\
(2005)^{(65) \star}\end{array}$ & 25 & $\mathrm{~F}$ & 0 & $\sim$ & $21-49$ & $\begin{array}{c}\text { LF }(25 \% \mathrm{E} \text { from fat, } \\
\quad \downarrow 2.5 \mathrm{MJ} / \mathrm{d})\end{array}$ & & $36 \cdot 3$ & $\sim$ & $\sim$ & $43 \cdot 8$ & 43.7 & $\mathrm{BIA}$ & 6.8 & $\sim$ & $\sim$ & $\sim$ & 33.5 & $\sim$ \\
\hline $\begin{array}{l}\text { Arvidsson et al. } \\
(2004)^{(66) \star}\end{array}$ & 20 & $\mathrm{~F}$ & 0 & 35 & $21-49$ & $\begin{array}{l}\mathrm{MF}(40-45 \% \mathrm{E} \\
\text { from fat, } \downarrow \\
2.5 \mathrm{MJ} / \mathrm{d})\end{array}$ & 10 weeks & 37.6 & $\sim$ & 0.95 & $45 \cdot 9$ & $\sim$ & BIA & 7.4 & $\sim$ & NSt & NSt & 22.9 & NS \\
\hline $\begin{array}{l}\text { Arvidsson et al. } \\
(2004)^{(66) \star}\end{array}$ & 20 & $\mathrm{~F}$ & 0 & 35 & $21-49$ & $\begin{array}{l}\text { LF }(20-25 \% \\
\quad E \text { from fat, } \\
\downarrow 22.5 \mathrm{MJ} / \mathrm{d})\end{array}$ & & 36.6 & $\sim$ & 0.94 & 44.5 & $\sim$ & BIA & 7.5 & $\sim$ & NSt & NSt & 33.2 & NS \\
\hline $\begin{array}{l}\text { Wood et al. } \\
(2006)^{(64) *}\end{array}$ & 14 & M & 100 & $\sim$ & $20-69$ & LC + fibre & 12 weeks & 29.7 & $\sim$ & $\sim$ & $32 \cdot 1$ & $\sim$ & DXA & 8.0 & $19.6 \dagger$ & $3.1 \dagger$ & NSt & $\sim$ & $\sim$ \\
\hline $\begin{array}{l}\text { Wood et al. } \\
(2006)^{(64) \star}\end{array}$ & 15 & M & 100 & $\sim$ & $20-69$ & LC + placebo & & 29.7 & $\sim$ & $\sim$ & $32 \cdot 1$ & $\sim$ & DXA & 8.0 & $16 \cdot 7 \dagger$ & $13.9 \dagger$ & NS† & $\sim$ & $\sim$ \\
\hline $\begin{array}{l}\text { Noakes et al. } \\
(2005)^{(67) *}\end{array}$ & 48 & $\mathrm{~F}$ & 0 & 49 & $20-65$ & $\begin{array}{l}\text { High-carbohydrate/LF } \\
\text { (64/20\% } \mathrm{E} \\
\text { isoenergetic) }\end{array}$ & 12 weeks & 33.0 & $\sim$ & $\sim$ & $\sim$ & 41.9 & DXA & 8.0 & $16 \cdot 7$ & $\sim$ & $\sim$ & $\sim$ & $\sim$ \\
\hline $\begin{array}{l}\text { Noakes et al. } \\
(2005)^{(67) *}\end{array}$ & 52 & $\mathrm{~F}$ & 0 & 50 & $20-65$ & $\begin{array}{l}\mathrm{HP} / \mathrm{LF}(34 / 20 \% \mathrm{E}, \\
\text { isoenergetic) }\end{array}$ & & $32 \cdot 0$ & $\sim$ & $\sim$ & $\sim$ & $42 \cdot 1$ & DXA & 8.7 & $25 \cdot 8$ & $\sim$ & $\sim$ & $\sim$ & $\sim$ \\
\hline $\begin{array}{l}\text { Pereira et al. } \\
(2004)^{(68) \star}\end{array}$ & 17 & $\mathrm{M} / \mathrm{F}$ & 24 & 33 & $18-40$ & $\begin{array}{l}\mathrm{LF}(6 \cdot 3 \mathrm{MJ} / \mathrm{d}, 18 \% \\
\quad \mathrm{E} \text { from fat) }\end{array}$ & $69 d$ & $\sim$ & $\sim$ & $\sim$ & 38.4 & $\sim$ & DXA & 10.5 & $5 \cdot 1 \dagger$ & $\sim$ & $\sim$ & $\sim$ & $\sim$ \\
\hline $\begin{array}{l}\text { Pereira et al. } \\
(2004)^{(68) \star}\end{array}$ & 22 & $\mathrm{M} / \mathrm{F}$ & 23 & 29 & $18-40$ & $\begin{array}{l}\text { Low GL (6.3 MJ/d, } \\
30 \% \mathrm{E} \text { from fat) }\end{array}$ & $65 d$ & $\sim$ & $\sim$ & $\sim$ & 39.4 & $\sim$ & DXA & 10.5 & $47.7 \dagger$ & $\sim$ & $\sim$ & $\sim$ & $\sim$ \\
\hline Mean \% WL & & & & & & & & & & & & & & 7.9 & & & & & \\
\hline
\end{tabular}

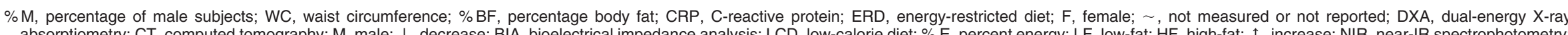
absorptiometry; CT, computed tomography; M, male; $\downarrow$, decrease; BIA, bieelectrical impedance analysis; LCD, low-calorie diet; \% E, percent energy; LF, low-fat; HF, high-fat; $\uparrow$, increase; NIR, near-IR spectrophotometry; $\mathrm{ER}$, energy restriction; MF, medium-fat; HP, high-protein; GL, glycaemic load.

Studies containing more

tMedian values or values calted.

from median values.

mass loss. 
$(\text { Table } 2)^{(11,69-72)}$. The types of physical activity intervention used were generally classified as exercise training, resistance training or aerobic exercise training. Based on mean BMI values, it would appear that the majority of studies ( $n$ 4) were carried out in overweight subjects. However, only one of these studies solely included overweight subjects ${ }^{(69)}$, with the other studies including both overweight and obese individuals ${ }^{(11,70-72)}$.

Overall, few studies $(n 5)$ used physical activity to induce weight loss, which did not exceed $5 \%(3 \mathrm{~kg})$ in any study. This is to be expected, as to achieve a weight loss of approximately $2 \mathrm{lb}(1 \mathrm{~kg})$ per week, a deficit of $4.2 \mathrm{MJ}$ (1000 kcal) would require walking or running $65-70$ miles $(105-113 \mathrm{~km})$ per week with energy intake remaining constant. Therefore, due to the smaller amount of weight loss observed, resulting changes in ORIM are also somewhat inconsistent. For example, TNF- $\alpha$ was shown to significantly decrease by $16 \%$ (after 12 weeks) in one study $^{(71)}$, decrease by $83 \%$ (after 5 months) in another study ${ }^{(70)}$ and significantly increase by $12 \%$ (after 1 year) in a third intervention ${ }^{(11)}$. Differences in baseline anthropometry, FM or percentage weight loss do not seem to explain the different effects of physical activity on TNF- $\alpha$ evident in these studies. However, differing intensities and/ or frequencies of exercise training in each study, or simply the duration of intervention, may partly explain the observed findings.

Another important consideration when using physical activity interventions as a method of weight loss is the role of fat-free mass (FFM). It is well documented that physical activity increases, or at least maintains, levels of $\mathrm{FFM}^{(73)}$, which is a likely explanation for the relatively smaller levels of total weight loss noted in these studies in comparison with the dietary interventions. Indeed, in those studies reporting FFM pre- and post-intervention ( $n 3$ ), it was seen to significantly increase by $5 \%$ after resistance training ${ }^{(69)}$ or remain unchanged (i.e. was conserved) after general exercise training ${ }^{(70,71)}$ in females. This finding agrees with a recent study by Hunter et al. who showed that females involved in resistance training maintained levels of FFM more than those involved in aerobic exercise or no exercise training at all ${ }^{(74)}$.

\section{Diet and physical activity interventions}

Interventions combining both diet and physical activity modifications ( $n$ 18) ranged in duration from 6 weeks to 2 years and the number of participants ranged from nineteen

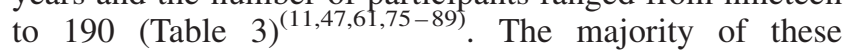
interventions were carried out in obese subjects $(50 \%)$, with $22 \%$ of studies in overweight and $11 \%$ in morbidly obese subjects. The mean baseline BMI value was not reported in three studies.

All but one of these studies ( $n$ 17) reported significant weight loss post-intervention ${ }^{(11,47,61,76-89)}$ and although not reported as absolute weight loss $(\mathrm{kg})$, the remaining study did report significant decreases in BMI and FM postintervention ${ }^{(75)}$. Similar to those interventions using dietary modification alone, a weight loss of at least $10 \%$ resulted in the greatest improvements in ORIM concentrations ${ }^{(80-83,85-88)}$. Studies reporting less than $10 \%$ weight

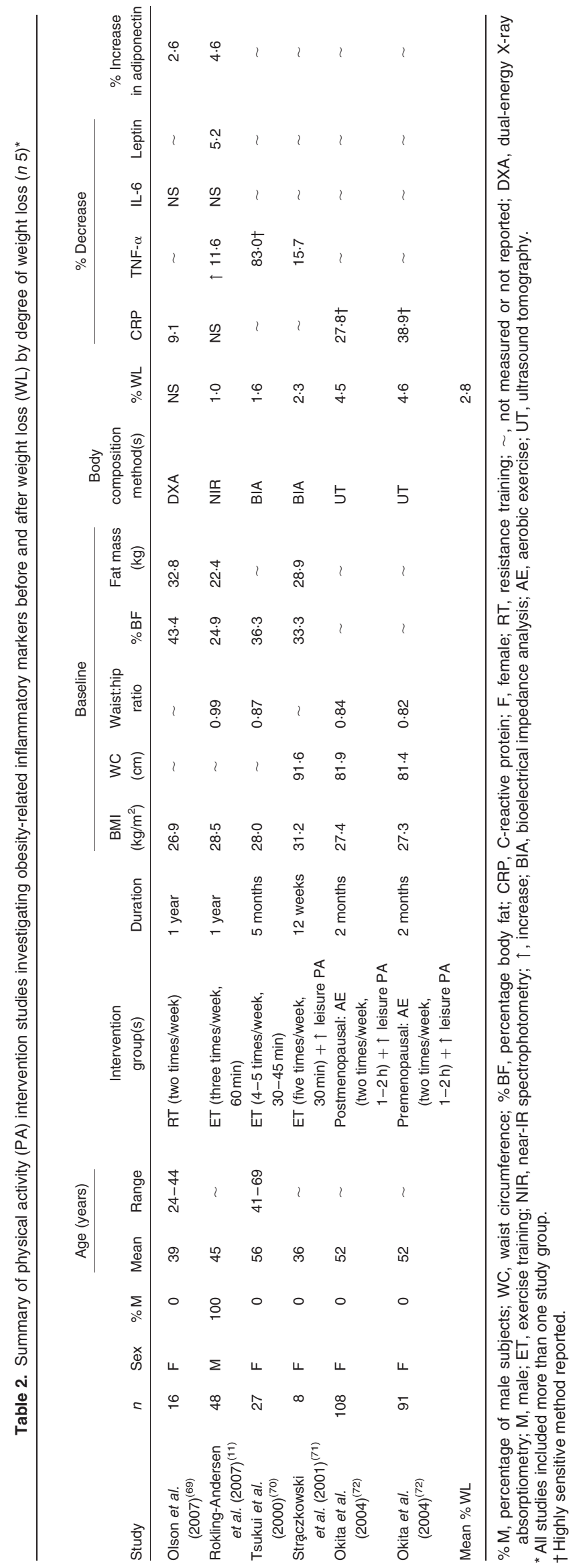




\section{䱏 NS Nutrition Research Reviews}

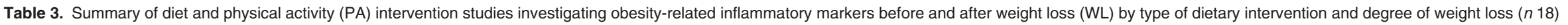

\begin{tabular}{|c|c|c|c|c|c|c|c|c|c|c|c|c|c|c|c|c|c|c|c|c|}
\hline \multirow[b]{2}{*}{ Study } & \multirow[b]{2}{*}{$n$} & \multirow[b]{2}{*}{ Sex } & \multirow[b]{2}{*}{$\% \mathrm{M}$} & \multicolumn{2}{|c|}{ Age (years) } & \multicolumn{2}{|c|}{ Intervention groups } & \multirow[b]{2}{*}{ Duration } & \multicolumn{5}{|c|}{ Baseline } & \multirow{2}{*}{$\begin{array}{l}\text { Body } \\
\text { composition } \\
\text { method(s) }\end{array}$} & \multirow[b]{2}{*}{$\% \mathrm{WL}$} & \multicolumn{4}{|c|}{$\%$ Decrease } & \multirow[b]{2}{*}{$\begin{array}{l}\% \text { Increase } \\
\text { in adiponectin }\end{array}$} \\
\hline & & & & Mean & Range & Diet & Lifestyle & & $\begin{array}{c}\mathrm{BMI} \\
\left(\mathrm{kg} / \mathrm{m}^{2}\right)\end{array}$ & $\begin{array}{l}\mathrm{WC} \\
(\mathrm{cm})\end{array}$ & $\begin{array}{l}\text { Waist:hip } \\
\text { ratio }\end{array}$ & $\% B F$ & $\begin{array}{c}\text { Fat } \\
\text { mass (kg) }\end{array}$ & & & CRP & TNF- $\alpha$ & IL-6 & Leptin & \\
\hline \multicolumn{21}{|l|}{ ERD } \\
\hline $\begin{array}{l}\text { Itoh et al. } \\
(2002)^{(75) *}\end{array}$ & 71 & $\mathrm{~F}$ & 0 & 46 & $\sim$ & $\operatorname{ERD}(\downarrow 2.1 \mathrm{MJ} / \mathrm{d})$ & Walking (40 min/d) & 3 months & 28.3 & $\sim$ & $\sim$ & $\sim$ & 26.5 & $\mathrm{BIA}+\mathrm{MRI}$ & $\sim$ & $\sim$ & $\sim$ & $\sim$ & $27 \cdot 6$ & $\sim$ \\
\hline $\begin{array}{l}\text { Sheu et al. } \\
(2008)^{(76) *}\end{array}$ & 21 & $\mathrm{~F}$ & 0 & 34 & $\sim$ & $\operatorname{ERD}(\downarrow 2 \cdot 1-4 \cdot 2 \mathrm{MJ} / \mathrm{d})$ & $\uparrow \mathrm{PA}$, not restricted & 12 weeks & 32.5 & $96 \cdot 3$ & 0.87 & $\sim$ & $\sim$ & $\sim$ & $5 \cdot 1$ & $48.9 \dagger$ & NS & NS & $17 \cdot 2$ & 33.9 \\
\hline $\begin{array}{l}\text { Ryan \& Nicklas } \\
(2004)^{(77) \star}\end{array}$ & 37 & $\mathrm{~F}$ & 0 & 58 & $50-70$ & $\operatorname{ERD}(\downarrow 1 \cdot 1-1 \cdot 2 \mathrm{MJ} / \mathrm{d})$ & $\begin{array}{l}\text { PA (three times/week, } \\
\quad 45 \mathrm{~min} \text { ) }\end{array}$ & 6 months & $\sim$ & $\sim$ & $\sim$ & $46 \cdot 8$ & $40 \cdot 2$ & $\mathrm{DXA}+\mathrm{CT}$ & $7 \cdot 1$ & $7 \cdot 0$ & NSt & $15.9 \dagger$ & $\sim$ & $\sim$ \\
\hline $\begin{array}{l}\text { Dvořáková- } \\
\text { Lorenzová } \\
\text { et al. }(2006)^{(78) \star}\end{array}$ & 40 & $\mathrm{~F}$ & 0 & $\sim$ & $25-35$ & ERD ( $\downarrow$ saturated fat) & $\begin{array}{l}\mathrm{AE} \text { (six times/week, } \\
60 \mathrm{~min})\end{array}$ & 9 weeks & 31.5 & 93.2 & 0.82 & $\sim$ & $\sim$ & MRI & 7.7 & $30.1 \dagger$ & $\sim$ & NS & $\sim$ & NS \\
\hline You et al. $(2004)^{(47) \star ~}$ & 17 & $\mathrm{~F}$ & 0 & 59 & $50-70$ & $\begin{array}{l}\text { ERD (to achieve WL } \\
\text { of } 0.5-1 \mathrm{~kg} / \text { week })\end{array}$ & $\begin{array}{l}\text { Treadmill (three times/ } \\
\text { week, } 20-60 \mathrm{~min} \text { ) }\end{array}$ & 6 months & $\sim$ & $\sim$ & 0.82 & $45 \cdot 6$ & 38.4 & $\mathrm{DXA}+\mathrm{CT}$ & 8.5 & 34.1 & 6.4 & $27 \cdot 1$ & $\sim$ & $\sim$ \\
\hline $\begin{array}{l}\text { Salas-Salvadó } \\
\text { et al. }(2006)^{(79) *}\end{array}$ & 19 & $M / F$ & 32 & 41 & $29-60$ & $\begin{array}{l}\text { ERD (3.3 MJ/d, } \\
13.5 \% \mathrm{E} \text { from fat) }\end{array}$ & PA $(60 \mathrm{~min} / \mathrm{d})$ & 6 weeks & 48.4 & $133 \cdot 6$ & 0.99 & 49.2 & $\sim$ & BIA & 9.0 & 35.5 & NS & $32 \cdot 7$ & $\sim$ & $\sim$ \\
\hline $\begin{array}{l}\text { Church et al. } \\
(2005)^{(80)}\end{array}$ & 71 & $M / F$ & 32 & 47 & $\sim$ & ERD & $\uparrow \mathrm{PA}$ & 9 months & 43.0 & 126.5 & $\sim$ & $\sim$ & $\sim$ & $\sim$ & 11.4 & $39.7 \dagger$ & $\sim$ & $\sim$ & $\sim$ & $\sim$ \\
\hline $\begin{array}{l}\text { Esposito et al. } \\
(2004)^{(81) \star}\end{array}$ & 55 & M & 100 & 44 & $35-55$ & $\begin{array}{l}\text { ERD (7.1-8.0 MJ/d, } \\
<30 \% \text { E from fat) }\end{array}$ & $\begin{array}{l}\uparrow \mathrm{PA}+\text { behaviour } \\
\text { modification }\end{array}$ & 2 years & 36.9 & $\sim$ & 1.02 & $\sim$ & $\sim$ & $\sim$ & $14 \cdot 6$ & $42.4 \dagger$ & $\sim$ & $31 \cdot 1 \dagger$ & $\sim$ & $\sim$ \\
\hline $\begin{array}{l}\text { Esposito et al. } \\
(2003)^{(82) \star}\end{array}$ & 60 & $\mathrm{~F}$ & 0 & 34 & $20-46$ & $\begin{array}{l}\text { ERD (5.4-6.3 MJ/d, } \\
<30 \% \text { E from fat) }\end{array}$ & $\begin{array}{l}\uparrow \mathrm{PA}+\text { behaviour } \\
\text { modification }\end{array}$ & 2 years & 35.0 & $\sim$ & 0.86 & $\sim$ & $\sim$ & $\sim$ & 14.7 & $34 \cdot 4 \uparrow \ddagger$ & $\sim$ & $32 \cdot 6+\ddagger$ & $\sim$ & 48.2 \\
\hline $\begin{array}{l}\text { St-Onge et al. } \\
(2008)^{(83) \star}\end{array}$ & 190 & $\mathrm{~F}$ & 0 & $\sim$ & $20-41$ & $\begin{array}{l}\text { Energy balance } \\
\text { before and after } \\
\text { active WL }\end{array}$ & PA (three times/week) & $>8$ weeks & 28.3 & 86.9 & $\sim$ & 44.8 & $\sim$ & $\mathrm{DXA}+\mathrm{CT}$ & 14.9 & $9.5 \dagger$ & NS† & 23.5 & $\sim$ & $\sim$ \\
\hline \multicolumn{21}{|l|}{ LCD } \\
\hline $\begin{array}{l}\text { Zahorska- } \\
\text { Markiewicz } \\
\text { et al. }(2000)^{(84)}\end{array}$ & 27 & $M / F$ & 15 & 48 & $\sim$ & LCD (4.2 MJ/d) & $\begin{array}{l}\text { PA + behaviour } \\
\text { counselling }\end{array}$ & 3 months & $36 \cdot 3$ & 99.0 & 0.89 & 42.5 & $40 \cdot 3$ & BIA & 9.6 & $\sim$ & $21.8 \dagger$ & $\sim$ & $\sim$ & $\sim$ \\
\hline $\begin{array}{l}\text { Ziccardi } e t \text { al. } \\
(2002)^{\left({ }^{(85) \times S}\right.}\end{array}$ & 56 & $\mathrm{~F}$ & 0 & 35 & $25-44$ & LCD (5.4 MJ/d) & $\begin{array}{l}\text { MD approach, PA (three } \\
\text { times/week, } 60 \mathrm{~min} \text { ) }\end{array}$ & 12 months & 37.2 & $\sim$ & 0.84 & $\sim$ & $\sim$ & $\sim$ & $\geq 10$ & $\sim$ & 31.0 & 46.5 & $\sim$ & $\sim$ \\
\hline $\begin{array}{l}\text { Nicoletta et al. } \\
(2003)^{(86) \times 5}\end{array}$ & 20 & $\mathrm{~F}$ & 0 & 37 & $25-44$ & LCD $(5.4 \mathrm{MJ} / \mathrm{d})$ & $\begin{array}{l}\text { MD approach, PA (three } \\
\text { times/week, } 60 \mathrm{~min} \text { ) }\end{array}$ & 1 year & 37.2 & $\sim$ & 0.85 & $\sim$ & $\sim$ & $\sim$ & $\geq 10$ & $\sim$ & 29.1 & $\sim$ & $\sim$ & $\sim$ \\
\hline $\begin{array}{l}\text { Marfella et al. } \\
(2004)^{(87) *}\end{array}$ & 67 & $\mathrm{~F}$ & 0 & 37 & $25-44$ & LCD $(5.4 \mathrm{MJ} / \mathrm{d})$ & $\begin{array}{l}\text { MD approach, PA (three } \\
\text { times/week, } 60 \mathrm{~min} \text { ) }\end{array}$ & 12 months & 37.6 & $\sim$ & 0.84 & $\sim$ & $\sim$ & $\sim$ & $\geq 10$ & $44 \cdot 1 \dagger$ & $31.0 \dagger$ & $61.9 \dagger$ & $\sim$ & $\sim$ \\
\hline $\begin{array}{l}\text { Dandona et al. } \\
(1998)^{(88) \star}\end{array}$ & 38 & $\mathrm{~F}$ & 0 & $\sim$ & $25-54$ & $\operatorname{LCD}(3.9-4.8 \mathrm{MJ} / \mathrm{d})$ & $\begin{array}{l}\mathrm{AE}(12-40 \mathrm{~min} / \mathrm{d})+ \\
\text { behaviour modification }\end{array}$ & $1-2$ years & 35.7 & $\sim$ & $\sim$ & $\sim$ & $\sim$ & $\sim$ & $12 \cdot 3$ & $\sim$ & 23.8 & $\sim$ & $\sim$ & $\sim$ \\
\hline $\begin{array}{l}\text { Mean \% WL } \\
\text { LF diets }\end{array}$ & & & & & & & & & & & & & & & $11 \cdot 0$ & & & & & \\
\hline $\begin{array}{l}\text { Obisesan et al. } \\
(2004)^{(89) \star}\end{array}$ & 61 & $M / F$ & 44 & 59 & $50-75$ & $\begin{array}{l}\mathrm{LF}(<30 \% \\
\quad \mathrm{E} \text { from fat) }\end{array}$ & $\begin{array}{l}\mathrm{PA} \text { (three times/week) }+ \\
45-60 \mathrm{~min} \text { walk/week }\end{array}$ & 24 weeks & $\sim$ & $\sim$ & $\sim$ & $\sim$ & 34.7 & DXA & 1.9 & 14.4 & $\sim$ & $\sim$ & $\sim$ & $\sim$ \\
\hline $\begin{array}{l}\text { Brekke et al. } \\
(2005)^{(61) *}\end{array}$ & 25 & $M / F$ & 72 & 42 & $25-55$ & $\begin{array}{l}\mathrm{LF}(\leq 30 \% \\
\quad \mathrm{E} \text { from fat) }\end{array}$ & $\begin{array}{l}\uparrow \mathrm{PA}(4-5 \text { times/week, } \\
30 \mathrm{~min})\end{array}$ & 16 weeks & $26 \cdot 0$ & $91 \cdot 2$ & $\sim$ & 29.5 & $\sim$ & $\mathrm{BIA}$ & $2 \cdot 1$ & $\sim$ & $\sim$ & $\sim$ & NS & NS \\
\hline $\begin{array}{l}\text { Rokling-Andersen } \\
\text { et al. }(2007)^{(11){ }_{\star}}\end{array}$ & 58 & M & 100 & 45 & $\sim$ & $\begin{array}{l}\text { LF (current } \\
\quad \text { recommendations) }\end{array}$ & $\begin{array}{l}\text { ET (three times/week, } \\
60 \mathrm{~min} \text { ) }\end{array}$ & 1 year & 28.4 & $\sim$ & 0.99 & $24 \cdot 1$ & 22.0 & $\mathrm{BIA}$ & $6 \cdot 3$ & NS & $2 \cdot 0$ & NS & 24.0 & NS \\
\hline Mean \%WL & & & & & & & & & & & & & & & 3.4 & & & & & \\
\hline
\end{tabular}

$\% \mathrm{M}$, percentage of male subjects; WC, waist circumference; \% BF, percentage body fat; CRP, C-reactive protein; ERD, energy-restricted diet; F, female; $\downarrow$, decrease; , not measured or not reported; BIA, bioelectrical impedance analysis; $\uparrow$, increase; DXA, dual-energy X-ray absorptiometry; CT, computed tomography; AE, aerobic exercise; M, male; E \%, percent energy; LCD, low-calorie diet; MD, multidisciplinary; LF, low-fat; ET, exercise training.

Studies including more than one study group.

t Highly sensitive method reported.

‡ Values calculated from median values.
$\S$ In addition to the diet and PA intervention, liposuction was performed in fifteen ${ }^{(85)}$ and twenty ${ }^{(86)}$ subjects, respectively, at least 3 months before plasma assays 
loss show inconsistent effects on the concentrations of ORIM $^{(11,47,61,76-79,84,89)}$. For example, after 6 months, Ryan \& Nicklas observed significant improvements in plasma CRP and IL- 6 concentrations ( 7 and $16 \%$ decrease, respectively), with no significant change in plasma TNF- $\alpha$ in thirty-seven postmenopausal females ${ }^{(77)}$, whereas You et al. ${ }^{(47)}$ found that plasma CRP, IL- 6 and TNF- $\alpha$ concentrations decreased by 34,27 and $6 \%$, respectively, in seventeen postmenopausal females with a similar degree of weight loss $(7-9 \% \text { or } 6-7 \mathrm{~kg})^{(47)}$. Subjects in the latter study ${ }^{(47)}$ lost slightly more FM compared with those in the study of Ryan \& Nicklas ${ }^{(77)}$, which may partly explain the discrepant ORIM results.

Only four out of the eighteen diet and physical activity studies investigated the effects of weight loss on circulating leptin concentrations ${ }^{(11,61,75,76)}$. Unfortunately, weight loss (kg) was not quantified in all studies, so meaningful comparisons cannot be drawn. However, an improvement in circulating leptin concentration was associated with a significant decrease in body fat, expressed as percentage change or absolute change ${ }^{(11,75,76)}$ and where the intervention failed to decrease FM despite a significant weight loss, circulating leptin concentration remained unchanged from that at baseline ${ }^{(61)}$.

Three of the diet and physical activity intervention studies that resulted in the greatest weight loss were also the longest in duration (1-2 years) and observed significant improvements in all ORIM measured ${ }^{(82,87,88)}$. The decrease in serum TNF- $\alpha$ concentrations ranged from $24 \%^{(88)}$ to $31 \%^{(87)}$, and the decrease in serum CRP and IL- 6 concentrations ranged from $34-44 \%$ and $33-62 \%$, respectively ${ }^{(82,87)}$. Moreover, serum adiponectin concentration was also shown to increase by $48 \%{ }^{(82)}$. These three studies were carried out in females; however, similar improvements in serum CRP and IL-6 (42 and $31 \%$, respectively) were also observed in men in a separate intervention ${ }^{(81)}$. Unfortunately in these four studies, only the simple anthropometric measures, BMI and/or waist:hip ratio were used; therefore, associations between inflammation, weight loss and adiposity cannot truly be identified as FM was not measured directly. One advantage, however, is that these four studies clearly demonstrate the benefit of long-term diet and lifestyle interventions in terms of the assessment of changes in inflammation following weight loss.

\section{Surgical interventions}

Subjects who underwent surgery for weight loss (Table 4$)^{(90-114)}$ were either obese $(20 \%)$ or morbidly obese $(80 \%)$. The duration of follow-up was longer than in other weight-loss interventions, ranging from 3 months to 3 years, and the number of participants in each study ( $n$ 25) ranged from seven to 106. Methods of surgical intervention were broadly classified into three types; fat removal surgery, gastric restrictive surgery or gastric bypass surgery, with some studies using either restrictive or bypass. In the majority of studies including morbidly obese patients ( $n 16)$, post-operative mean BMI was significantly reduced to below $40 \mathrm{~kg} / \mathrm{m}^{2}$ and in the two studies with the greatest overall weight loss ${ }^{(108,109)}$, post-operative BMI dramatically fell to below $30 \mathrm{~kg} / \mathrm{m}^{2}$.
A greater range in weight loss $(3-50 \%$ or $3-84 \mathrm{~kg})$ was observed in the surgical interventions, with the gastric bypass surgery leading to a greater mean weight loss $(33 \%)$ than the fat removal, or restrictive surgery ( 7 and $27 \%$, respectively). Results for most ORIM are more consistent compared with those observed in the dietary interventions, with or without physical activity. Significant reductions in circulating CRP concentrations were reported in fifteen out of nineteen studies and ranged from 17 to $79 \%$. The decrease in leptin ( $n$ 15) ranged from 15 to $76 \%$ and the increase in adiponectin ( $n$ 10) ranged from 13 to $209 \%$. Overall, improvements in circulating IL-6 and TNF- $\alpha$ concentrations are somewhat less consistent than those observed for the other ORIM. IL-6 concentration was not significantly decreased in five out of twelve studies reporting its concentration post-operatively and in those that do report significant decreases, the effects are smaller (9 to $59 \%$ ) compared with those seen for other ORIM. Serum TNF- $\alpha$ was only significantly decreased in one out of seven studies, despite this being the study reporting the smallest degree of weight loss ${ }^{(90)}$.

A study by Busetto et al. ${ }^{(92)}$ observed no significant effects on CRP or IL-6, a small but significant decrease in leptin $(23 \%)$ and a significant decrease in adiponectin concentration $(11 \%)$ at 6 months, effects associated with a modest weight loss of $9 \%(9 \mathrm{~kg})$ post-surgery. This was the only study reviewed which used large-volume ultrasoundassisted megalipoplasty as the method of surgery (which mainly removes subcutaneous fat) and the only one to assess the immediate post-operative effects, following subjects at 1, 3, 28 and $180 \mathrm{~d}$ post-surgery. Subjects also had a lower BMI at baseline $\left(37.5 \mathrm{~kg} / \mathrm{m}^{2}\right)$ relative to those in the other studies. Although body weight was significantly reduced at each time-point, FM only decreased after $3 \mathrm{~d}$ and remained at this level up to day 180. An immediate inflammatory response was observed $1 \mathrm{~d}$ post-surgery, characterised by a dramatic increase in leptin, CRP and IL-6, and a decrease in adiponectin concentrations. However, the authors concluded that as patients recovered from surgery, levels of the ORIM returned to baseline values within 6 months, with only the concentration of leptin and adiponectin remaining significantly reduced post-surgery ${ }^{(92)}$. The importance of adequate duration of follow-up of surgical interventions to allow for recovery and stabilisation of inflammation will be explored in more detail later in the present review.

As mentioned above, surgical interventions that included insulin-resistant or diabetic subjects, or those with cardiovascular risk factors, were not excluded from the present review. The majority of patients presenting for gastric surgery are morbidly obese and, consequently, it is common that additional risk factors or co-morbidities of obesity are present. In all of the surgical intervention studies reviewed, measures of disease risk were studied in parallel with ORIM. Overall, weight loss was coupled with improvements in metabolic risk factors $(93,98,110)$, insulin resistance and/or insulin sensitivity $(90,92,95,96,98,99,103,105-109,111,112,114)$ and a decrease in cardiovascular $\operatorname{risk}^{(90,95-97,99-102,104,106,107,109,112-114)}$. Moreover, improvements in glucose tolerance were reported as a reversal of type 2 diabetes, with subjects reverting back to impaired glucose tolerance or, in some cases, achieving a 
WS Nutrition Research Reviews

Table 4. Summary of surgical intervention studies investigating obesity-related inflammatory markers before and after weight loss (WL) by surgical procedure and degree of weight loss ( $n$ 25)

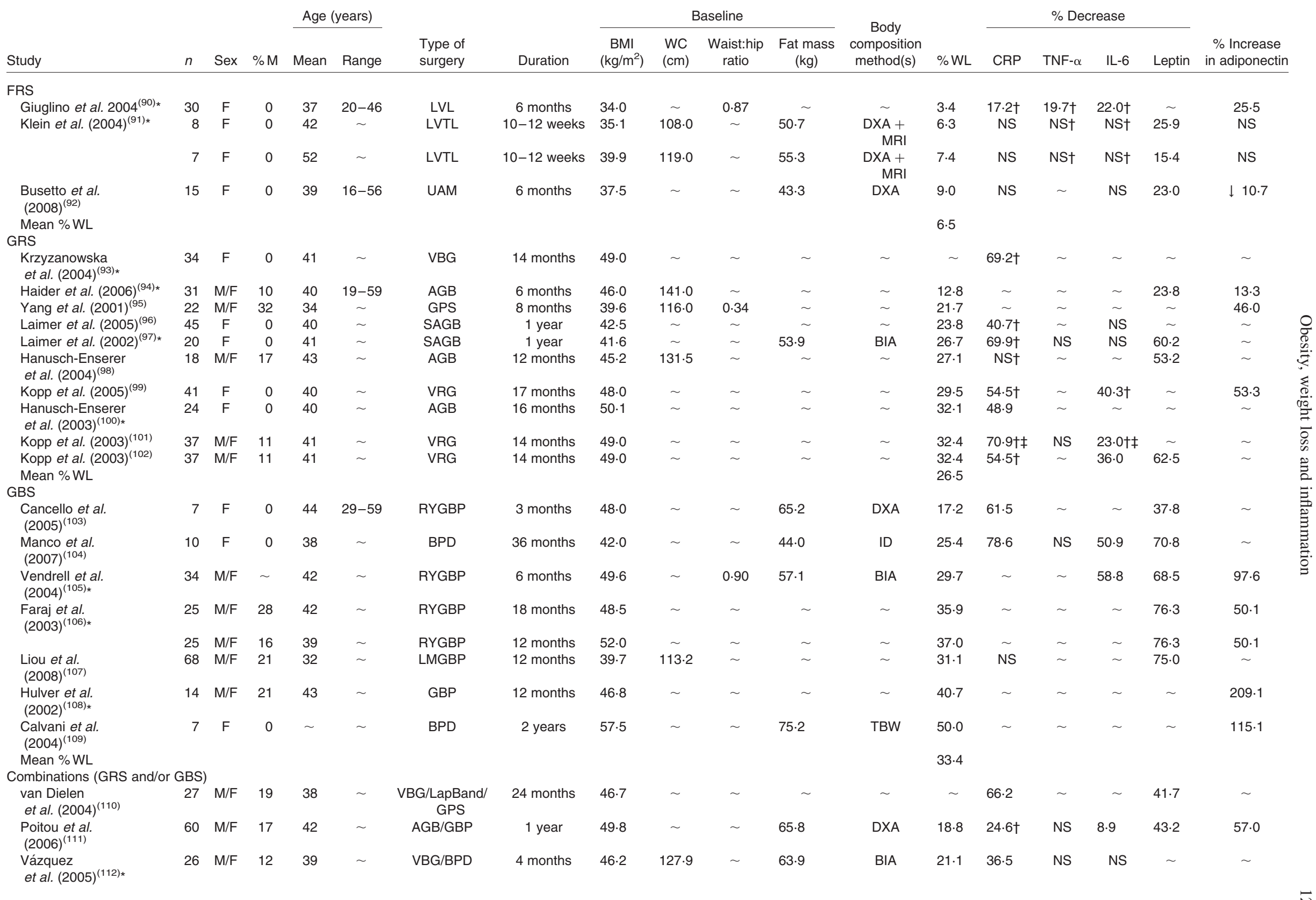




\section{Weight maintenance}

When weight loss is achieved through diet and physical activity modifications, the body experiences an unusual physiological state of negative energy balance. This can in turn disrupt a number of processes within the body, including inflammation. Consequently, the majority of weight-loss interventions discussed are limited in their design since very few ( $n$ 8) included a weight-maintenance phase before post-intervention sampling to allow for stabilisation of the body's inflammatory profile. However, half of these studies did not distinguish between the active weight-loss and maintenance phases of their interventions, only reporting results for the total study duration ${ }^{(47,55,56,83)}$. Therefore, it is unclear if differences in inflammatory profiles are evident between these phases. In the remaining studies, concentrations of ORIM immediately post-intervention and after weight maintenance were considered as different time-points ${ }^{(48,54,62,79)}$. Considine et al. showed an improvement in serum leptin concentrations (53\% decrease) following a $10 \%$ weight loss by LCD, although leptin concentrations showed a slight increase during the 4-week maintenance phase, albeit that it still remained significantly lower compared with that at baseline ${ }^{(54)}$. In contrast, Keogh et al. found that a 12-week energyrestricted diet was unable to improve CRP concentration and slightly increased adiponectin, although not significantly so. At 1-year follow-up, a small $6 \%(5 \mathrm{~kg})$ weight loss was maintained and did give rise to significantly greater adiponectin concentrations; however, the limitations of this study in terms of small sample size have been discussed previously $^{(48)}$. The effects of dietary manipulation on inflammation are clearly highlighted by Kasim-Karakas et al. ${ }^{(62)}$. During the initial phase of their intervention, subjects were placed on an energy-balanced diet that reduced fat content from $35 \%$ to $15 \%$ of energy over 4 months. Weight did not change during this phase, although energy intake was much greater than that at baseline (9.4 $v$. $6 \cdot 6 \mathrm{MJ} / \mathrm{d})$. This induced an acute inflammatory response with a significant increase in serum CRP and a significant decrease in serum adiponectin concentration. For the remaining 8 months, the second phase, subjects remained on a $15 \%$ fat ad libitum diet. Compared with that of the energy-balanced diet $(9 \cdot 4 \mathrm{MJ} / \mathrm{d})$, energy intake was reduced to $5 \cdot 2 \mathrm{MJ} / \mathrm{d}$ and a significant weight loss $(8 \%$ or $6 \mathrm{~kg})$ was also achieved. Although the serum concentrations of CRP, IL-6 and adiponectin were significantly improved following 
the acute response, they did not significantly differ from that at baseline ${ }^{(62)}$. Finally, Salas-Salvado et al. ${ }^{(79)}$ reported that a $9 \%(11 \mathrm{~kg})$ weight loss achieved by a 6-week LCD was associated with a significant improvement in plasma IL-6 and CRP concentrations. However, following a 2-week weight-maintenance period, plasma IL-6 increased slightly but remained significantly lower compared with baseline. Plasma CRP, on the other hand, rose sharply to a higher concentration than that at baseline. In this case, the authors were forced to conclude that the improvements in inflammatory markers were mainly attributable to the energy restriction during the 6-week LCD as these improvements were not sustained during weight maintenance $^{(79)}$. More recently it has been quantified that in order to maintain improvements in ORIM (CRP and adiponectin in this case), following a short-term (8 weeks) VLCD, a minimum weight loss of $10 \%$ must be maintained in the long term (3 years) ${ }^{(115)}$

The duration of follow-up needed in gastric surgery interventions is likely to be longer in comparison with diet and/or physical activity interventions, to allow for stabilisation of physiological processes and overall recovery postsurgery. Despite this, few studies ( $n$ 5) have included different time-points to determine changes in inflammatory profiles post-surgery. Faraj et al. ${ }^{(106)}$ classified subjects into two groups; weight-stable at 18 months and weight-reducing at 12 months (twenty-five subjects per group), with the latter group achieving more weight loss $(37 \%$ or $52 \mathrm{~kg} v .36 \%$ or $49 \mathrm{~kg}$ ) and at a greater rate per month (3 v. 1\%) than those in the former group. Unfortunately, improvements in ORIM were only quantified for the study group as a whole; therefore, differences between the groups in terms of their inflammatory profiles cannot be elucidated ${ }^{(106)}$. A recent intervention by Busetto et al. is the only study included in the present review that demonstrates the acute pro-inflammatory response immediately post-surgery ${ }^{(92)}$. At later time-points, responses observed for each ORIM are inconsistent. For example, at 6 months, CRP has been shown to remain unchanged from baseline ${ }^{(92,100,110)}$ or slightly higher, although not significantly so ${ }^{(107)}$. Furthermore, at 12 months, plasma CRP concentration has been reported as slightly increased $^{(107)}$, unchanged ${ }^{(98)}$, or remaining lower at 24 months ${ }^{(110)}$. Overall, if generalised to all ORIM, the evidence suggests that at least 2 years is required post-surgery for stabilisation of the inflammatory profile. In all of these surgical interventions, significant weight loss was achieved at each time-point up to 1 year ${ }^{(92,98,107,110)}$ and although van Dielen et al. did not report absolute weight loss in $\mathrm{kg}$, BMI and percentage total excess weight loss were significantly lower at each time-point up to 2 years $^{(110)}$. However, more recently, weight regain has been reported up to 5 years postsurgery, suggesting that follow-ups of a longer duration are required, especially when investigating the effects on inflammation $^{(116)}$

\section{Hidden dangers}

When investigating associations between adiposity, inflammation and the effects of weight loss, measures of body composition - whether indirect (using BMI) or direct (measuring FM or percentage body fat) - are crucial.
However, as mentioned previously, metabolic differences have been observed between SAT and VAT, with VAT in particular associated with a greater metabolic risk $^{(24-29)}$. Although waist circumference and waist:hip ratio have proved effective as surrogate measures of body fat distribution $^{(30,117-119)}$, advances in techniques, such as MRI or computed tomography scans, have allowed researchers to accurately distinguish the different fat depots.

Seven out of eight studies included in the present review $^{(47,56,75,77,78,83,91)}$ directly measured the VAT and/or SAT areas using MRI or computed tomography scans before and after weight loss (the other study used ultrasound tomography to quantify fat in the abdominal wall $\left.{ }^{(72)}\right)$. Circulating levels of $\mathrm{CRP}^{(56,72,78)}$ and leptin ${ }^{(75)}$ were shown to be positively correlated with intra-abdominal VAT in women, similar to findings (CRP only) previously reported in men ${ }^{(120)}$. In most cases, significant reductions in total body weight (range 5-16\%) resulted in significant percentage losses of VAT in females $(47,56,75,77,78,83)$ which were greater than that of SAT, when reported. In the majority of studies, this was also associated with significant improvements in ORIM (Tables 1-4). In addition, changes in VAT and plasma IL-6 following significant weight loss were shown to be independent predictors of changes in insulin sensitivity ${ }^{(77)}$. Unfortunately, no studies reviewed in the present paper quantified VAT and SAT areas in males. Salas-Salvadó et al. ${ }^{(79)}$ failed to show a positive association between SAT ORIM production (determined from biopsies) and systemic inflammation after weight loss. The authors speculated that the VAT depot may have, in part, attributed to the improved inflammatory profile observed although not accounted for in this study ${ }^{(79)}$. In support of this, Klein et al. showed that abdominal liposuction, which mainly removed SAT, was unable to produce improvements in the ORIM measured ${ }^{(91)}$. A recent systematic review of sixty-one studies, including more than 2000 measured changes in VAT and SAT, found a favourable reduction in VAT (rather than SAT) with modest weight loss. Furthermore, this effect was lessened as weight loss increased and it was concluded that this finding may shed some light on the mechanisms underlying the beneficial effects associated with modest weight loss ${ }^{(121)}$. Although VAT initially contributes to an improved inflammatory profile following modest weight loss, the general pattern highlighted throughout this review may suggest that SAT (and total adipose tissue loss) also has a role in modulating changes in inflammation with further weight loss.

\section{Discussion}

In the present review we have described the effects of weight loss on a number of ORIM in overweight and obese, but otherwise healthy, populations. A total of sixty-six intervention studies were included, grouped by the strategy used to induce weight loss, with each study measuring one or more ORIM.

\section{Effects of weight loss}

The overall conclusion from the present and previous reviews $^{(44-46)}$ is that weight loss does improve inflammation in terms of a number of ORIM, specifically 
characterised by a decrease in the inflammatory markers (CRP, TNF- $\alpha$, IL-6 and leptin) and an increase in the antiinflammatory marker, adiponectin. Moreover, the greatest and more consistent improvements are noted in those studies achieving at least a $10 \%$ weight loss. A systematic review by Selvin et al. quantified that a $1 \mathrm{~kg}$ weight loss will produce a $-0.13 \mathrm{mg} / \mathrm{l}$ change in CRP through diet and lifestyle modifications, which is increased to a $-0.16 \mathrm{mg} / \mathrm{l}$ change per $1 \mathrm{~kg}$ loss of body weight induced by gastric surgery ${ }^{(46)}$. It remains to be elucidated whether similar changes per $1 \mathrm{~kg}$ weight loss would be observed for the other ORIM.

Overall, LCD (with or without lifestyle modifications) and gastric bypass surgery appeared to result in the greatest average weight loss and, therefore, the greatest improvements in ORIM compared with the other dietary or surgical methods, respectively. Where two or more diets of different macronutrient composition were compared, no differences between diets were apparent with the exception of a low-glycaemic-load diet $v$. a low-fat diet, where the former resulted in a marked decrease in serum CRP concentration compared with the latter. However, it has previously been concluded that more dietary interventions focusing on specific macronutrients and their effects on inflammation are needed to determine any mechanistic differences $^{(45,122)}$

Physical activity interventions, although fewer in number, did not produce consistent improvements in ORIM; however, this is most probably due to the small, or lack of significant degrees of weight loss observed in these studies. Indeed, in three studies where diet and/or physical activity intervention groups were compared, it was concluded that diet plus physical activity led to the greatest improvements in some ${ }^{(11,61)}$ if not all ${ }^{(47)}$ ORIM measured compared with the diet and/or physical activity groups individually.

\section{Methodological issues}

A number of limitations concerning study design referred to throughout the present review may mask associations between weight loss and inflammation.

Sex. The majority of studies ( $n$ 42) have been carried out only in female subjects. In the studies that have included males (usually in a much smaller proportion compared with females), effects of weight loss on inflammation have not been analysed for each sex individually and therefore do not allow for any sex-specific differences to be determined. This finding suggests that women are generally a more obvious target group for weight-loss interventions and given the fact that differences in ORIM do exist between males and females (for example, CRP reportedly higher in females $^{(123,124)}$, and that adiposity is associated with inflammation to a greater extent in females $\left.{ }^{(124)}\right)$, it seems possible that this target group is potentially more appealing for researchers.

Age. In general, subjects ranged in age between 32 and 61 years (where reported), with some studies including quite a large age range, for example, $20-65$ years $^{(67)}$. It is well known that both adiposity and inflammation increase with age, so combining both young and middle-aged adults represents another potential limitation within the literature. Furthermore, the hormonal status of women should be indicated in studies as it has been shown that postmenopausal women have increased inflammation ${ }^{(125,126)}$. Only one study included in the present review (a physical activity intervention) split their group of females by menopausal status; however, no differences in the amount of weight loss, or effects on ORIM, were observed between the groups ${ }^{(72)}$.

Sample size. The number of participants in the majority of studies ranged from seven to seventy-one, and those that included more than one study group contained relatively fewer subjects per group in most cases. Only three studies contained more than 100 subjects $^{(72,83,113)}$. The limitation of small sample size has been noted in a number of studies, some only finding significant improvements in ORIM when results for different intervention groups are pooled for $\operatorname{analysis}^{(48,53,66)}$

Duration. Some of the null or inconsistent findings reported in the present review may, in part, be attributed to the short duration of these studies. The effects of shortterm weight loss, i.e. 4-6 weeks (or less), on inflammation are conflicting and prompt the question: are the positive effects observed due to weight loss, or solely because of the energy restriction (Fig. 1)? Few studies $(n 8)$ in the present review include a period of weight maintenance after intervention and, where included, normalisation of ORIM is evident, in some cases levels reverting back to that at baseline or higher, despite a lower weight being maintained. Furthermore, where physical activity was used as a method of weight loss, with or without dietary modification, only ten out of twenty-three studies took into account the stabilisation of the acute inflammatory effects of exercise.

Analysis of obesity-related inflammatory markers. The most common technique used for the analysis of ORIM by studies included in the present review is the ELISA. However, a number of other commercially available assays have also been reported, including chemiluminescent assays $^{(50)}$, nephelometric latex immunoassays ${ }^{(51,63,72)}$ and RIA $^{(54,57,66,75,76)}$. While techniques for ORIM assessment continue to improve in sensitivity and specificity there is no accepted 'gold standard' method for assessment. Furthermore, interpretation of data is hampered by the paucity of data on what is considered a normal concentration of such molecules. In addition, several ORIM, including TNF- $\alpha$, are likely to act in an autocrine or paracrine manner, mediating their inflammatory effect at a local cellular level and therefore, systemic concentrations of such molecules may not provide an accurate reflection of their inflammatory potential.

Assessment of body composition. The way in which studies report weight changes after intervention lacks consistency. For example, some report weight changes in absolute $(\mathrm{kg})$ terms and others only report the relative losses (\%). Therefore, future studies should report weight loss, both in absolute $(\mathrm{kg})$ and relative (\%) terms, in order to determine if it is the weight loss relative to an 
individual's starting point (\%) or the extent of the energy deficit $(\mathrm{kg})$ that is important in terms of improving inflammatory status. BMI (i.e. body weight adjusted for height to classify overweight and obesity) ${ }^{(1)}$ and waist circumference (to take account of central obesity and provide an estimate of body fat distribution $)^{(30,117-119)}$ are widely used anthropometric tools in research. However, obesity itself is defined as excess body fat, levels of which cannot be determined using these surrogate measures, as they do not distinguish between FM and FFM. Approximately half of the studies included in the present review used only BMI, waist circumference and/or waist:hip ratio, which impedes clarification of the relationship between adiposity, weight loss and inflammation. Given the fact that obesity-related inflammation is due to increasing adiposity, as excess fat is stored in adipocytes, failure to directly measure FM means that these studies cannot truly identify the associations between inflammation and adiposity, both before, and after, weight loss. In studies where FM was measured, bioelectrical impedance analysis and dual-energy X-ray absorptiometry were the two most common methods used. However, a recent study has demonstrated that these methods may not be reliable in detecting changes in body composition following weight loss ${ }^{(127)}$. These studies also fail to take account of the proportions of FM and FFM in excess weight (i.e. 75 and $25 \%$, respectively) $)^{(128)}$ and, therefore, the relationship between the FM:FFM ratio and ORIM has also not been investigated. Furthermore, expressing FM as a percentage of total body weight (i.e. percentage body fat) has more recently been criticised within the literature, being described as a 'fallacy' ${ }^{\text {(129) }}$. In order to take account of the individual variations in FM and FFM, alternative indices, first described over 15 years $\mathrm{ago}^{(130)}$, have been proposed where FM $(\mathrm{kg})$ and FFM $(\mathrm{kg})$ are individually adjusted for height (i.e. divided by height in meters squared) to give FM index and FFM index respectively ${ }^{(131-133)}$. The fact that these indices are not considered by any intervention study included in the present review suggests how little they have been applied in research over the last decade. We have recently highlighted the importance of using these indices when examining the association between adiposity and inflammation by demonstrating that FM index is more strongly associated with serum CRP levels in females ( $\leq 30$ years) compared with FM in absolute terms, with percentage body fat showing no significant association ${ }^{(134)}$.

\section{Conclusion}

Overall, the present review indicates that during a period of weight loss, the unfavourable inflammatory profile associated with increased adiposity can be improved, thus providing further evidence for the beneficial effects of weight loss in overweight and obesity in terms of reducing the risk of co-morbidities. However, it is likely that the improvements observed are mainly due to a negative energy balance in the short term, rather than decreasing adiposity. More randomised, controlled intervention studies are required to accurately determine the time needed, in which a reduced weight is maintained, in order to benefit from improved inflammatory status longterm. Furthermore, it is recommended that these studies include younger adults, with equal numbers of both men and women, and contain measures of body composition appropriately adjusted for body size.

\section{Acknowledgements}

The present review was supported by funding from the Department of Education and Learning (DEL). We would also like to give special thanks to Tracy McCaffrey and Carol Wilson for their comments on the drafts of the manuscript, and to Kilian McDaid for graphic design.

There are no conflicts of interest.

\section{References}

1. World Health Organization (2000) Obesity: Preventing and Managing the Global Epidemic, no. 894. Geneva: WHO.

2. Craig R \& Mindell J (2008) Health Survey for England 2006 Latest Trends. London: The Information Centre.

3. Wardle J \& Boniface D (2008) Changes in the distributions of body mass index and waist circumference in English adults, 1993/1994 to 2002/2003. Int J Obes (Lond) 32, $527-532$.

4. McPherson K, Marsh T \& Brown M (2007) Modelling future trends in obesity and the impact on health. http:// www.foresight.gov.uk

5. Hotamisligil GS, Shargill NS \& Spiegelman BM (1993) Adipose expression of tumor necrosis factor- $\alpha$ : direct role in obesity-linked insulin resistance. Science 259, 87-91.

6. Trayhurn P (2005) The biology of obesity. Proc Nutr Soc 64, $31-38$.

7. Hauner H (2005) Secretory factors from human adipose tissue and their functional role. Proc Nutr Soc 64, 163-169.

8. Zhang Y, Proenca R, Maffei M, et al. (1994) Positional cloning of the mouse obese gene and its human homologue. Nature 372, 425-432.

9. Mohamed-Ali V, Pinkney JH \& Coppack SW (1998) Adipose tissue as an endocrine and paracrine organ. Int J Obes Relat Metab Disord 22, 1145-1158.

10. Moreno-Aliaga MJ, Campión J, Milagro FI, et al. (2005) Adiposity and proinflammatory state: the chicken or the egg. Adipocytes 1, 1-16.

11. Rokling-Andersen MH, Reseland JE, Veierod MB, et al. (2007) Effects of long-term exercise and diet intervention on plasma adipokine concentrations. Am J Clin Nutr 86, 1293-1301.

12. Scherer PE, Williams S, Fogliano M, et al. (1995) A novel serum protein similar to $\mathrm{Clq}$, produced exclusively in adipocytes. J Biol Chem 270, 26746-26749.

13. Yudkin JS, Stehouwer CD, Emeis JJ, et al. (1999) C-reactive protein in healthy subjects: associations with obesity, insulin resistance, and endothelial dysfunction: a potential role for cytokines originating from adipose tissue? Arterioscler Thromb Vasc Biol 19, 972-978.

14. Trayhurn P \& Wood IS (2004) Adipokines: inflammation and the pleiotropic role of white adipose tissue. Br J Nutr 92, 347-355.

15. Greenberg AS \& Obin MS (2006) Obesity and the role of adipose tissue in inflammation and metabolism. Am J Clin Nutr 83, 461S-465S.

16. Das UN (2001) Is obesity an inflammatory condition? Nutrition 17, 953-966. 
17. Engstrom G, Hedblad B, Stavenow L, et al. (2003) Inflammation-sensitive plasma proteins are associated with future weight gain. Diabetes 52, 2097-2101.

18. Fantuzzi G (2005) Adipose tissue, adipokines, and inflammation. J Allergy Clin Immunol 115, 911-919.

19. Hotamisligil G (2006) Inflammation and metabolic disorders. Nature 444, 860-867.

20. Ahima RS \& Flier JS (2000) Adipose tissue as an endocrine organ. Trends Endocrinol Metab 11, 327-332.

21. Festa A, D'Agostino R Jr, Williams K, et al. (2001) The relation of body fat mass and distribution to markers of chronic inflammation. Int $J$ Obes Relat Metab Disord 25, 1407-1415.

22. Cottam DR, Mattar SG, Barinas-Mitchell E, et al. (2004) The chronic inflammatory hypothesis for the morbidity associated with morbid obesity: implications and effects of weight loss. Obes Surg 14, 589-600.

23. Wellen KE \& Hotamisligil GS (2005) Inflammation, stress, and diabetes. $J$ Clin Invest 115, 1111-1119.

24. Hartz AJ, Rupley DC \& Rimm AA (1984) The association of girth measurements with disease in 32,856 women. Am J Epidemiol 119, 71-80.

25. Ohlson LO, Larsson B, Svardsudd K, et al. (1985) The influence of body fat distribution on the incidence of diabetes mellitus. 13.5 years of follow-up of the participants in the study of men born in 1913. Diabetes 34, 1055-1058.

26. Rimm EB, Stampfer MJ, Giovannucci E, et al. (1995) Body size and fat distribution as predictors of coronary heart disease among middle-aged and older US men. Am J Epidemiol 141, 1117-1127.

27. Rexrode KM, Carey VJ, Hennekens CH, et al. (1998) Abdominal adiposity and coronary heart disease in women. JAMA 280, 1843-1848.

28. Janssen I, Katzmarzyk PT \& Ross R (2002) Body mass index, waist circumference, and health risk: evidence in support of current National Institutes Of Health guidelines. Arch Intern Med 162, 2074-2079.

29. von Eyben FE, Mouritsen E, Holm J, et al. (2003) Intraabdominal obesity and metabolic risk factors: a study of young adults. Int J Obes Relat Metab Disord 27, 941-949.

30. Snijder MB, van Dam RM, Visser M, et al. (2006) What aspects of body fat are particularly hazardous and how do we measure them? Int J Epidemiol 35, 83-92.

31. Fain JN (2006) Release of interleukins and other inflammatory cytokines by human adipose tissue is enhanced in obesity and primarily due to the nonfat cells. Vitam Horm 74, 443-477.

32. Hirsch J \& Batchelor B (1976) Adipose tissue cellularity in human obesity. Clin Endocrinol Metab 5, 299-311.

33. Powell K (2007) Obesity: the two faces of fat. Nature 447, $525-527$.

34. de Ferranti S \& Mozaffarian D (2008) The perfect storm: obesity, adipocyte dysfunction, and metabolic consequences. Clin Chem 54, 945-955.

35. Spalding KL, Arner E, Westermark PO, et al. (2008) Dynamics of fat cell turnover in humans. Nature 453, $783-787$.

36. Frayn KN (2005) Obesity and metabolic disease: is adipose tissue the culprit? Proc Nutr Soc 64, 7-13.

37. Weisberg SP, McCann D, Desai M, et al. (2003) Obesity is associated with macrophage accumulation in adipose tissue. J Clin Invest 112, 1796-1808.

38. Xu H, Barnes GT, Yang Q, et al. (2003) Chronic inflammation in fat plays a crucial role in the development of obesity-related insulin resistance. J Clin Invest 112, $1821-1830$.
39. Fain JN, Madan AK, Hiler ML, et al. (2004) Comparison of the release of adipokines by adipose tissue, adipose tissue matrix, and adipocytes from visceral and subcutaneous abdominal adipose tissues of obese humans. Endocrinology 145, 2273-2282.

40. Bassuk SS, Rifai N \& Ridker PM (2004) High-sensitivity C-reactive protein: clinical importance. Curr Probl Cardiol 29, 439-493.

41. Lyon CJ, Law RE \& Hsueh WA (2003) Minireview: adiposity, inflammation, and atherogenesis. Endocrinology 144, 2195-2200.

42. Nawrocki AR \& Scherer PE (2004) The delicate balance between fat and muscle: adipokines in metabolic disease and musculoskeletal inflammation. Curr Opin Pharmacol 4 , 281-289.

43. Ronti T, Lupattelli G \& Mannarino E (2006) The endocrine function of adipose tissue: an update. Clin Endocrinol (Oxf) 64, 355-365.

44. Dietrich M \& Jialal I (2005) The effect of weight loss on a stable biomarker of inflammation, C-reactive protein. Nutr Rev 63, 22-28.

45. Devaraj S, Kasim-Karakas S \& Jialal I (2006) The effect of weight loss and dietary fatty acids on inflammation. Curr Atheroscler Rep 8, 477-486.

46. Selvin E, Paynter NP \& Erlinger TP (2007) The effect of weight loss on C-reactive protein: a systematic review. Arch Intern Med 167, 31-39.

47. You T, Berman DM, Ryan AS, et al. (2004) Effects of hypocaloric diet and exercise training on inflammation and adipocyte lipolysis in obese postmenopausal women. J Clin Endocrinol Metab 89, 1739-1746.

48. Keogh JB, Brinkworth GD \& Clifton PM (2007) Effects of weight loss on a low-carbohydrate diet on flow-mediated dilatation, adhesion molecules and adiponectin. $\mathrm{Br} J \mathrm{Nutr}$ 98, 852-859.

49. Davì G, Guagnano MT, Ciabattoni G, et al. (2002) Platelet activation in obese women: role of inflammation and oxidant stress. JAMA 288, 2008-2014.

50. McLaughlin T, Abbasi F, Lamendola C, et al. (2002) Differentiation between obesity and insulin resistance in the association with C-reactive protein. Circulation 106, 2908-2912.

51. Bougoulia M, Triantos A \& Koliakos G (2006) Effect of weight loss with or without orlistat treatment on adipocytokines, inflammation, and oxidative markers in obese women. Hormones (Athens) 5, 259-269.

52. Heilbronn LK, Noakes M \& Clifton PM (2001) Energy restriction and weight loss on very-low-fat diets reduce C-reactive protein concentrations in obese, healthy women. Arterioscler Thromb Vasc Biol 21, 968-970.

53. Hannum SM, Carson L, Evans EM, et al. (2004) Use of portion-controlled entrees enhances weight loss in women. Obes Res 12, 538-546.

54. Considine RV, Sinha MK, Heiman ML, et al. (1996) Serum immunoreactive-leptin concentrations in normal-weight and obese humans. N Eng J Med 334, 292-295.

55. Bruun JM, Verdich C, Toubro S, et al. (2003) Association between measures of insulin sensitivity and circulating levels of interleukin-8, interleukin-6 and tumor necrosis factor- $\alpha$. Effect of weight loss in obese men. Eur $J$ Endocrinol 148, 535-542.

56. Tchernof A, Nolan A, Sites CK, et al. (2002) Weight loss reduces C-reactive protein levels in obese postmenopausal women. Circulation 105, 564-569.

57. Clément K, Viguerie N, Poitou C, et al. (2004) Weight loss regulates inflammation-related genes in white adipose tissue of obese subjects. FASEB $J \mathbf{1 8}, 1657-1669$. 
58. Bastard JP, Jardel C, Bruckert E, et al. (2000) Elevated levels of interleukin 6 are reduced in serum and subcutaneous adipose tissue of obese women after weight loss. J Clin Endocrinol Metab 85, 3338-3342.

59. Raitakari M, Ilvonen T, Ahotupa M, et al. (2004) Weight reduction with very-low-caloric diet and endothelial function in overweight adults: role of plasma glucose. Arterioscler Thromb Vasc Biol 24, 124-128.

60. Heald AH, Golding C, Sharma R, et al. (2004) A substitution model of dietary manipulation is an effective means of optimising lipid profile, reducing C-reactive protein and increasing insulin-like growth factor-1. $\mathrm{Br} J$ Nutr 92, 809-818.

61. Brekke HK, Lenner RA, Taskinen MR, et al. (2005) Lifestyle modification improves risk factors in type 2 diabetes relatives. Diabetes Res Clin Pract 68, 18-28.

62. Kasim-Karakas SE, Tsodikov A, Singh U, et al. (2006) Responses of inflammatory markers to a low-fat, highcarbohydrate diet: effects of energy intake. Am J Clin Nutr 83, 774-779.

63. O'Brien KD, Brehm BJ, Seeley RJ, et al. (2005) Dietinduced weight loss is associated with decreases in plasma serum amyloid a and C-reactive protein independent of dietary macronutrient composition in obese subjects. J Clin Endocrinol Metab 90, 2244-2249.

64. Wood RJ, Volek JS, Davis SR, et al. (2006) Effects of a carbohydrate-restricted diet on emerging plasma markers for cardiovascular disease. Nutr Metab 3, 19.

65. Viguerie N, Vidal H, Arner P, et al. (2005) Adipose tissue gene expression in obese subjects during low-fat and highfat hypocaloric diets. Diabetologia 48, 123-131.

66. Arvidsson E, Viguerie N, Andersson I, et al. (2004) Effects of different hypocaloric diets on protein secretion from adipose tissue of obese women. Diabetes 53, 1966-1971.

67. Noakes M, Keogh JB, Foster PR, et al. (2005) Effect of an energy-restricted, high-protein, low-fat diet relative to a conventional high-carbohydrate, low-fat diet on weight loss, body composition, nutritional status, and markers of cardiovascular health in obese women. Am J Clin Nutr 81, 1298-1306.

68. Pereira MA, Swain J, Goldfine AB, et al. (2004) Effects of a low-glycemic load diet on resting energy expenditure and heart disease risk factors during weight loss. JAMA 292, 2482-2490.

69. Olson TP, Dengel DR, Leon AS, et al. (2007) Changes in inflammatory biomarkers following one-year of moderate resistance training in overweight women. Int J Obes (Lond) 31, 996-1003.

70. Tsukui S, Kanda T, Nara M, et al. (2000) Moderateintensity regular exercise decreases serum tumor necrosis factor- $\alpha$ and HbA1c levels in healthy women. Int $J$ Obes Relat Metab Disord 24, 1207-1211.

71. Strączkowski M, Kowalska I, Dzienis-Straczkowska S, et al. (2001) Changes in tumor necrosis factor- $\alpha$ system and insulin sensitivity during an exercise training program in obese women with normal and impaired glucose tolerance. Eur J Endocrinol 145, 273-280.

72. Okita K, Nishijima H, Murakami T, et al. (2004) Can exercise training with weight loss lower serum C-reactive protein levels? Arterioscler Thromb Vasc Biol 24, 1868-1873.

73. Ballor DL \& Poehlman ET (1994) Exercise-training enhances fat-free mass preservation during diet-induced weight loss: a meta-analytical finding. Int $\mathrm{J}$ Obes Relat Metab Disord 18, 35-40.
74. Hunter GR, Byrne NM, Sirikul B, et al. (2008) Resistance training conserves fat-free mass and resting energy expenditure following weight loss. Obesity 16, 1045-1051.

75. Itoh K, Imai K, Masuda $\mathrm{T}$, et al. (2002) Relationship between changes in serum leptin levels and blood pressure after weight loss. Hypertens Res 25, 881-886.

76. Sheu WH, Chang T, Lee W, et al. (2008) Effect of weight loss on proinflammatory state of mononuclear cells in obese women. Obesity 16, 1033-1038.

77. Ryan AS \& Nicklas BJ (2004) Reductions in plasma cytokine levels with weight loss improve insulin sensitivity in overweight and obese postmenopausal women. Diabetes Care 27, 1699-1705.

78. Dvořáková-Lorenzová $\mathrm{A}$, Suchanek $\mathrm{P}$, Havel $\mathrm{PJ}$, et al. (2006) The decrease in C-reactive protein concentration after diet and physical activity induced weight reduction is associated with changes in plasma lipids, but not interleukin-6 or adiponectin. Metabolism 55, 359-365.

79. Salas-Salvadó J, Bullo M, Garcia-Lorda P, et al. (2006) Subcutaneous adipose tissue cytokine production is not responsible for the restoration of systemic inflammation markers during weight loss. Int J Obes (Lond) 30, 1714-1720.

80. Church TS, Willis MS, Priest EL, et al. (2005) Obesity, macrophage migration inhibitory factor, and weight loss. Int J Obes (Lond) 29, 675-681.

81. Esposito K, Giugliano F, Di Palo C, et al. (2004) Effect of lifestyle changes on erectile dysfunction in obese men: a randomized controlled trial. JAMA 291, 2978-2984.

82. Esposito K, Pontillo A, Di Palo C, et al. (2003) Effect of weight loss and lifestyle changes on vascular inflammatory markers in obese women. JAMA 289, 1799-1804.

83. St-Onge M, Desmond R, Hunter G, et al. (2008) Baseline inflammatory markers do not modulate the lipid response to weight loss. Metabolism 57, 598-604.

84. Zahorska-Markiewicz B, Janowska J, OlszaneckaGlinianowicz M, et al. (2000) Serum concentrations of TNF- $\alpha$ and soluble TNF- $\alpha$ receptors in obesity. Int J Obes Relat Metab Disord 24, 1392-1395.

85. Ziccardi P, Nappo F, Giugliano G, et al. (2002) Reduction of inflammatory cytokine concentrations and improvement of endothelial functions in obese women after weight loss over one year. Circulation 105, 804-809.

86. Nicoletti G, Giugliano G, Pontillo A, et al. (2003) Effect of a multidisciplinary program of weight reduction on endothelial functions in obese women. J Endocrinol Invest 26, R5-R8.

87. Marfella R, Esposito K, Siniscalchi M, et al. (2004) Effect of weight loss on cardiac synchronization and proinflammatory cytokines in premenopausal obese women. Diabetes Care 27, 47-52.

88. Dandona P, Weinstock R, Thusu K, et al. (1998) Tumor necrosis factor- $\alpha$ in sera of obese patients: fall with weight loss. J Clin Endocrinol Metab 83, 2907-2910.

89. Obisesan TO, Leeuwenburgh C, Phillips T, et al. (2004) C-reactive protein genotypes affect baseline, but not exercise training-induced changes, in C-reactive protein levels. Arterioscler Thromb Vasc Biol 24, 1874-1879.

90. Giugliano G, Nicoletti G, Grella E, et al. (2004) Effect of liposuction on insulin resistance and vascular inflammatory markers in obese women. Br J Plast Surg 57, 190-194.

91. Klein S, Fontana L, Young VL, et al. (2004) Absence of an effect of liposuction on insulin action and risk factors for coronary heart disease. $N$ Engl J Med 350, 2549-2557.

92. Busetto L, Bassetto F, Zocchi M, et al. (2008) The effects of the surgical removal of subcutaneous adipose tissue on 
energy expenditure and adipocytokine concentrations in obese women. Nutr Metab Cardiovasc Dis 18, 112-120.

93. Krzyzanowska K, Mittermayer F, Kopp HP, et al. (2004) Weight loss reduces circulating asymmetrical dimethylarginine concentrations in morbidly obese women. J Clin Endocrinol Metab 89, 6277-6281.

94. Haider DG, Schindler K, Schaller G, et al. (2006) Increased plasma visfatin concentrations in morbidly obese subjects are reduced after gastric banding. J Clin Endocrinol Metab 91, 1578-1581.

95. Yang W, Lee W, Funahashi T, et al. (2001) Weight reduction increases plasma levels of an adipose-derived antiinflammatory protein, adiponectin. J Clin Endocrinol Metab 86, 3815-3819.

96. Laimer M, Kaser S, Kranebitter M, et al. (2005) Effect of pronounced weight loss on the nontraditional cardiovascular risk marker matrix metalloproteinase-9 in middle-aged morbidly obese women. Int J Obes (Lond) 29, 498-501.

97. Laimer M, Ebenbichler CF, Kaser S, et al. (2002) Markers of chronic inflammation and obesity: a prospective study on the reversibility of this association in middle-aged women undergoing weight loss by surgical intervention. Int $J$ Obes Relat Metab Disord 26, 659-662.

98. Hanusch-Enserer U, Cauza E, Spak M, et al. (2004) Improvement of insulin resistance and early atherosclerosis in patients after gastric banding. Obes Res 12, 284-291.

99. Kopp HP, Krzyzanowska K, Mohlig M, et al. (2005) Effects of marked weight loss on plasma levels of adiponectin, markers of chronic subclinical inflammation and insulin resistance in morbidly obese women. Int J Obes (Lond) $\mathbf{2 9}$, 766-771.

100. Hanusch-Enserer U, Cauza E, Spak M, et al. (2003) Acutephase response and immunological markers in morbid obese patients and patients following adjustable gastric banding. Int J Obes Relat Metab Disord 27, 355-361.

101. Kopp HP, Hopp CW, Festa A, et al. (2003) Impact of weight loss on inflammatory proteins and their association with the insulin resistance syndrome in morbidly obese patients. Arterioscler Thromb Vasc Biol 23, 1042-1047.

102. Kopp CW, Kopp HP, Steiner S, et al. (2003) Weight loss reduces tissue factor in morbidly obese patients. Obes Res 11, 950-956.

103. Cancello R, Henegar C, Viguerie N, et al. (2005) Reduction of macrophage infiltration and chemoattractant gene expression changes in white adipose tissue of morbidly obese subjects after surgery-induced weight loss. Diabetes 54, 2277-2286.

104. Manco M, Fernandez-Real JM, Equitani F, et al. (2007) Effect of massive weight loss on inflammatory adipocytokines and the innate immune system in morbidly obese women. J Clin Endocrinol Metab 92, 483-490.

105. Vendrell J, Broch M, Vilarrasa N, et al. (2004) Resistin, adiponectin, ghrelin, leptin, and proinflammatory cytokines: relationships in obesity. Obes Res 12, 962-971.

106. Faraj M, Havel PJ, Phelis S, et al. (2003) Plasma acylationstimulating protein, adiponectin, leptin, and ghrelin before and after weight loss induced by gastric bypass surgery in morbidly obese subjects. J Clin Endocrinol Metab 88, 1594-1602.

107. Liou JM, Lin JT, Lee WJ, et al. (2008) The serial changes of ghrelin and leptin levels and their relations to weight loss after laparoscopic minigastric bypass surgery. Obes Surg 18, 84-89.

108. Hulver MW, Zheng D, Tanner CJ, et al. (2002) Adiponectin is not altered with exercise training despite enhanced insulin action. Am J Physiol Endocrinol Metab 283, E861-E865.
109. Calvani M, Scarfone A, Granato L, et al. (2004) Restoration of adiponectin pulsatility in severely obese subjects after weight loss. Diabetes 53, 939-947.

110. van Dielen FM, Buurman WA, Hadfoune M, et al. (2004) Macrophage inhibitory factor, plasminogen activator inhibitor-1, other acute phase proteins, and inflammatory mediators normalize as a result of weight loss in morbidly obese subjects treated with gastric restrictive surgery. J Clin Endocrinol Metab 89, 4062-4068.

111. Poitou C, Coussieu C, Rouault C, et al. (2006) Serum amyloid A: a marker of adiposity-induced low-grade inflammation but not of metabolic status. Obesity (Silver Spring) 14, 309-318.

112. Vázquez LA, Pazos F, Berrazueta JR, et al. (2005) Effects of changes in body weight and insulin resistance on inflammation and endothelial function in morbid obesity after bariatric surgery. $J$ Clin Endocrinol Metab 90, 316-322.

113. Gannagé-Yared M, Yaghi C, Habre B, et al. (2008) Osteoprotegerin in relation to body weight, lipid parameters, insulin sensitivity, adipocytokines, and C-reactive protein in obese and non-obese young individuals: results from both cross-sectional and interventional study. Eur $J$ Endocrinol 158, 353-359.

114. Botella-Carretero JI, Alvarez-Blasco F, MartinezGarcia MA, et al. (2007) The decrease in serum IL-18 levels after bariatric surgery in morbidly obese women is a time-dependent event. Obes Surg 17, 1199-1208.

115. Madsen EL, Rissanen A, Bruun JM, et al. (2008) Weight loss larger than $10 \%$ is needed for general improvement of levels of circulating adiponectin and markers of inflammation in obese subjects: a 3-year weight loss study. Eur $J$ Endocrinol 158, 179-187.

116. Magro DL, Geloneze B, Delfini R, et al. (2008) Long-term weight regain after gastric bypass: a 5 -year prospective study. Obes Surg 18, 648-651.

117. Lean ME, Han TS \& Morrison CE (1995) Waist circumference as a measure for indicating need for weight management. BMJ 311, 158-161.

118. Han TS, van Leer EM, Seidell JC, et al. (1995) Waist circumference action levels in the identification of cardiovascular risk factors: prevalence study in a random sample. BMJ 311, 1401-1405.

119. Anonymous (1998) Clinical Guidelines on the Identification, Evaluation, and Treatment of Overweight and Obesity in Adults - The Evidence Report. National Institutes of Health. Obes Res 6, Suppl. 2, 51S-209S.

120. Lemieux I, Pascot A, Prud'homme D, et al. (2001) Elevated C-reactive protein: another component of the atherothrombotic profile of abdominal obesity. Arterioscler Thromb Vasc Biol 21, 961-967.

121. Chaston TB \& Dixon JB (2008) Factors associated with percent change in visceral versus subcutaneous abdominal fat during weight loss: findings from a systematic review. Int $J$ Obes (Lond) 32, 619-628.

122. Clifton PM (2003) Diet and C-reactive protein. Curr Atheroscler Rep 5, 431-436.

123. Albert MA, Glynn RJ \& Ridker PM (2003) Plasma concentration of $\mathrm{C}$-reactive protein and the calculated Framingham Coronary Heart Disease Risk Score. Circulation 108, 161-165.

124. Thorand B, Baumert J, Döring A, et al. (2006) Sex differences in the relation of body composition to markers of inflammation. Atherosclerosis 184, 216-224.

125. Ridker PM, Hennekens CH, Rifai N, et al. (1999) Hormone replacement therapy and increased plasma concentration of C-reactive protein. Circulation 100, 713-716. 
126. Pradhan AD, Manson JE, Rossouw JE, et al. (2002) Inflammatory biomarkers, hormone replacement therapy, and incident coronary heart disease: prospective analysis from the Women's Health Initiative observational study. JAMA 288, 980-987.

127. Minderico CS, Silva AM, Keller K, et al. (2008) Usefulness of different techniques for measuring body composition changes during weight loss in overweight and obese women. Br J Nutr 99, 432-441.

128. Webster JD, Hesp R \& Garrow JS (1984) The composition of excess weight in obese women estimated by body density, total body water and total body potassium. Hum Nutr Clin Nutr 38, 299-306.

129. Cole TJ, Fewtrell MS \& Prentice A (2008) The fallacy of using percentage body fat as a measure of adiposity. Am $J$ Clin Nutr 87, 1959.
130. VanItallie TB, Yang M, Heymsfield SB, et al. (1990) Height-normalized indices of the body's fat-free mass and fat mass: potentially useful indicators of nutritional status. Am J Clin Nutr 52, 953-959.

131. Schutz Y, Kyle UU \& Pichard C (2002) Fat-free mass index and fat mass index percentiles in Caucasians aged 18-98 y. Int J Obes Relat Metab Disord 26, 953-960.

132. Kyle UG, Schutz Y, Dupertuis YM, et al. (2003) Body composition interpretation: contributions of the fat-free mass index and the body fat mass index. Nutrition 19, 597-604.

133. Kyle UG, Genton L, Gremion G, et al. (2004) Aging, physical activity and height-normalized body composition parameters. Clin Nutr 23, 79-88.

134. Forsythe LK, Livingstone MBE, Barnes MS, et al. (2008) C-reactive protein and body composition in a representative sample of young adults. Proc Nutr Soc (In the Press). 